\title{
Circular RNA CircEYA3 induces energy production to promote pancreatic ductal adenocarcinoma progression through the miR-1294/c-Myc axis
}

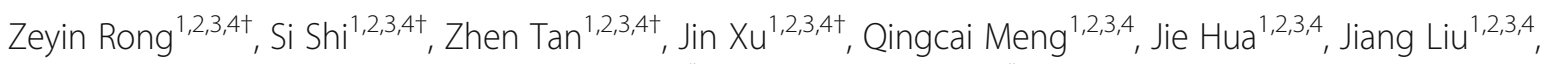
Bo Zhang ${ }^{1,2,3,4}$, Wei Wang ${ }^{1,2,3,4}$, Xianjun $Y u^{1,2,3,4^{*}}$ and Chen Liang ${ }^{1,2,3,4^{*}}$ (i)

\begin{abstract}
Background: Extensive studies have demonstrated the pivotal roles of circular RNAs (circRNAs) in the occurrence and development of different human cancers. However, the expression and regulatory roles of circRNAs in pancreatic ductal adenocarcinoma (PDAC) are unclear.

Methods: CircEYA3 was explored based on Gene Expression Omnibus (GEO) dataset analysis. qRT-PCR was applied to determine the expression of circRNAs, miRNAs and mRNAs in PDAC cells and tissues. The biological roles of circEYA3 in vitro and in vivo were determined by performing a series of functional experiments. Further, dual luciferase reporter, fluorescence in situ hybridization (FISH), RNA pull-down assays, and RNA immunoprecipitation (RIP) assays were used to confirm the interaction of circEYA3 with miR-1294.

Results: CircEYA3 was elevated in PDAC tissues and cells, and a higher level of circEYA3 was significantly associated with a poorer prognosis in patients with PDAC. Functionally, circEYA3 increased energy production via ATP synthesis to promote PDAC progression in vitro and in vivo. Mechanistically, circEYA3 functions as an endogenous miR-1294 sponge to elevate c-Myc expression, thus exerting its oncogenic functions.
\end{abstract}

Conclusion: CircEYA3 promotes the progression of PDAC through the miR-1294/c-Myc signalling axis, and circEYA3 may be an efficient molecular therapeutic target in PDAC.

Keywords: CircEYA3, miR-1294, C-Myc, ATP production, Pancreatic ductal adenocarcinoma

\section{Background}

The prognosis of pancreatic ductal adenocarcinoma (PDAC), a highly aggressive and lethal malignancy, remains unsatisfactory, with a 5 -year overall survival (OS) rate of less than $5 \%$ [1]. PDAC is going to have secondhighest global rate of cancer-associated mortality within

\footnotetext{
* Correspondence: yuxianjun@fudanpci.org; liangchen@fudanpci.org ${ }^{\dagger}$ Zeyin Rong, Si Shi, Zhen Tan and Jin Xu contributed equally to this work. 'Department of Pancreatic Surgery, Fudan University Shanghai Cancer Center, 270 Dong'An Road, Shanghai 200032, PR China

Full list of author information is available at the end of the article
}

the next decade [2]. Despite the numerous clinical endeavours directed at PDAC patients, the outcome of PDAC remains unfavourable due to its low rate of early diagnosis and is characteristic of high metastasis [3]. Hence, determining the potential mechanism of PDAC tumorigenesis and increasing the efficiency of early PDAC diagnosis are urgently needed to develop novel therapeutic strategies for this cancer.

Circular RNAs (circRNAs), an abundant RNA species, are an enigmatic group of noncoding RNAs (ncRNAs) formed by unique back-splicing events; they have a

(c) The Author(s). 2021 Open Access This article is licensed under a Creative Commons Attribution 4.0 International License, which permits use, sharing, adaptation, distribution and reproduction in any medium or format, as long as you give appropriate credit to the original author(s) and the source, provide a link to the Creative Commons licence, and indicate if changes were made. The images or other third party material in this article are included in the article's Creative Commons licence, unless indicated otherwise in a credit line to the material. If material is not included in the article's Creative Commons licence and your intended use is not permitted by statutory regulation or exceeds the permitted use, you will need to obtain permission directly from the copyright holder. To view a copy of this licence, visit http://creativecommons.org/licenses/by/4.0/ The Creative Commons Public Domain Dedication waiver (http://creativecommons.org/publicdomain/zero/1.0/) applies to the data made available in this article, unless otherwise stated in a credit line to the data. 
continuous closed structure without a $5^{\prime}$ end or $3^{\prime}$ polyadenylated tail and have become a popular topic in RNA research $[4,5]$. CircRNAs are acknowledged to be conserved across species and to be tissue- or developmental stage-specific, and they can resist exonuclease degradation [6]. In addition, circRNAs are translated and involved in the regulation of gene expression at both the transcriptional and translational levels [7]. Accumulating studies have shown that circRNAs are abnormally aberrantly expressed and participate in the pathogenesis of different cancers, such as lung cancer [8], gastric cancer [9], colorectal cancer [10], osteosarcoma [11], and melanoma [12]. In PDAC, some dysregulated circRNAs have been identified to play crucial roles in proliferation and progression. For example, according to previous reports, circBFAR acts as a miR-34b-5p sponge to facilitate the proliferation and metastasis of PDAC cells by activating MET signalling [13]. More recently, Shen et al. revealed that a negative feedback loop was formed between circNEIL3 and ADAR1 that can promote the progression of PDAC through the miR-432-5p/ADAR1/GLI1 axis [14]. To the best of our knowledge, although some dysregulated circRNAs have been reported to play crucial roles in the initiation and development of PDAC [15], here are still many unknowns that need exploration and study to elucidate the underlying mechanisms of circRNAs in PDAC.

In our present study, we focused on the upregulated circRNAs in PDAC based on GEO dataset analysis for the first time and characterized a novel oncogenic circRNA, circEYA3 (hsa_circ_0007895). CircEYA3 was derived from the EYA3 gene, exhibited an apparently elevated level in PDAC and was closely correlated with the prognosis of PDAC patients. Mechanistically, circEYA3 functioned as a sponge for has-miR-1294 (miR1294) to elevate the level of c-Myc. c-Myc is highly important in PDAC [16], and circEYA3 was found to exert its oncogenic functions by elevating ATP production in PDAC cells. Collectively, our findings indicated that circEYA3 may be a novel prognostic biomarker and promising therapeutic target for PDAC.

\section{Materials and methods}

Tissue specimens and cell lines

A total of 104 PDAC and matched non-cancerous tissues were collected from patients who underwent surgical resection without preoperative chemotherapy at Fudan University Shanghai Cancer Center (FUSCC), between 2013 and 2017. Each sample was evaluated by two independent pathologists. This study was approved by the Clinical Research Ethics Committee of FUSCC. Written informed consent was acquired from each patient before participation in this study. Human PDAC cells (Capan-1, MiaPaCa-2, SW1990, PANC-1, BXPC-3, and CFPAC-1) and human pancreatic ductal epithelial (HPDE) cells were obtained from ATCC. Cells were cultured as previously mentioned $[17,18]$.

\section{Immunohistochemical (IHC) analysis}

For IHC staining, slides were stained with antibodies against c-Myc (1:150, ab32072, Abcam), E-cadherin (1: 400, \#3195, CST), N-cadherin (1:50, \#13116, CST), Bax (1:400, \#14796, CST), cleaved caspase-3 (1:400, \#9661, CST), and Ki-67 (1:400, \#12202S, CST) following standard procedures previously described [17].

\section{RNA extraction, genomic DNA (gDNA) extraction, quantitative real-time PCR (qRT-PCR) analysis, RNase $R$ treatment and actinomycin $D$ (ActD) treatment}

Total RNA was extracted from PDAC cells, tissues and matched non-cancerous tissues with TRIzol Reagent (Invitrogen, USA) in line with the manufacturer's instructions. gDNA was isolated using a gDNA Reagent Kit (Sangon Biotech, Shanghai, China). cDNA was synthesized from $1 \mu \mathrm{g}$ of total RNA using a PrimeScript RT Reagent Kit (Accurate Biology, China). qRT-PCR was performed with a SYBR Green PCR Kit (Accurate Biology, China) in an ABI 7900HT System (Applied Biosystems, USA) to determine the expression levels of candidate genes. The relative circRNA/mRNA and miRNA expression levels were normalized to those of GAPDH and U6, respectively, by using the $2^{-\triangle \Delta C T}$ method. For RNase R treatment, $10 \mu \mathrm{g}$ of total RNA from the indicated cells was mixed with or without RNase $\mathrm{R}(3 \mathrm{U} / \mu \mathrm{g})$ for $20 \mathrm{~min}$ at $37^{\circ} \mathrm{C}$. Next, circEYA3 and linear EYA3 mRNA were reverse transcribed with specific primers and analysed by qRT-PCR or PCR followed by nucleic acid agarose gel electrophoresis. For ActD treatment, cells were exposed to $2 \mu \mathrm{g} / \mathrm{mL}$ ActD (Sigma) at the indicated times. Then, the expression of the circRNA or linear mRNA was detected and analysed using qRT-PCR. Each experiment was carried out at least three times. All primers used in the experiment were synthesized by NovaBio (Shanghai, China) and are listed Additional file 1:Table S1.

Oligonucleotides (oligos), plasmids and cell transfection Small interfering RNAs (siRNAs) targeting circEYA3 and the relevant control siRNA were synthesized by RiboBio (Guangzhou, China). SiRNAs targeting c-Myc were synthesized by NovaBio (Shanghai, China). The sequences of the siRNAs are shown in Additional file 1: Table S2. To construct the circEYA3 overexpression plasmid, the full-length cDNA sequence of human circEYA3 by RiboBio (Guangzhou, China) and inserted into the pLCDH-circ expression vector to construct the circEYA3 overexpression vector pLCDH-circEYA3. The mimics, inhibitor and corresponding negative controls 
(NCs) for miR-1294 were synthesized by and purchased from GenePharma (Shanghai, China). The siRNAs, miRNA mimics and miRNA inhibitor were transiently transfected into cells with Lipofectamine 3000 (Invitrogen, CA, USA). To construct cell lines with stable overexpression of circEYA3, HEK-293 T cells were transfected with the circEYA3 overexpression vector pLCDH-circEYA3 and the control vector pLCDH-circ along with the psPAX2 packaging plasmid and pMD2.G envelope plasmid with Lipofectamine 3000. Forty-eight hours later, lentivirus was harvested and transduced into PDAC cells, which were then selected with $3 \mu \mathrm{g} / \mathrm{mL}$ puromycin (Sangon Biotech, Shanghai, China) for 2 weeks to establish the stable overexpression cell line.

\section{Cell proliferation assays}

For the Cell Counting Kit-8 (CCK-8) assay, the treated PDAC cells were seeded into 96-well plates at a concentration of $3 \times 10^{3} /$ well. Then, $10 \mu \mathrm{L}$ of CCK- 8 assay solution (Dojindo, Japan) was added and incubated in the dark for $2 \mathrm{~h}$. The absorbance at $450 \mathrm{~nm}$ was measured every $24 \mathrm{~h}$ with a microplate reader (BioTek Instruments, USA). For the EdU incorporation assay, an EdU cell proliferation assay kit (Beyotime, China) was used to detect the proliferation of treated PDAC cells. In brief, treated cells $\left(100 \mu \mathrm{L}\right.$ of a $2 \times 10^{4}$ cells $/ \mathrm{mL}$ suspension) were seeded into 96-well plates, and $50 \mathrm{mM}$ EdU was added at $37^{\circ} \mathrm{C}$ for a 2 -h incubation. After fixation with $4 \%$ paraformaldehyde and permeabilization with $1 \%$ Triton $\mathrm{X}-100,100 \mu \mathrm{L}$ of Click reaction cocktail was added to the treated cells, and the cells were then stained with Hoechst 33342. Immunostaining was imaged and quantitatively evaluated with a fluorescence microscope (Olympus, Japan). For the colony formation assay, equal numbers of treated PDAC cells were inoculated into 6well plates and incubated at $37^{\circ} \mathrm{C}$ for 2 weeks. At the end of the incubation period, the cells were fixed with $4 \%$ paraformaldehyde and stained with $1 \%$ crystal violet. Then, macroscopic colonies were photographed and counted. All experiments were repeated three times.

\section{Western blot analysis}

In brief, proteins were isolated from PDAC cells and tumor tissues using RIPA buffer supplemented with proteinase and phosphatase inhibitors, and the protein concentration was determined with BCA reagent (Beyotime, China). Equal amounts of protein were electrophoresed on $10 \%$ SDS-PAGE gels and were then transferred onto PVDF membranes (Millipore), which were soaked for 2 $\mathrm{h}$ in $5 \%$ skim milk. Subsequently, the membranes were probed with primary antibodies specific for the following proteins: c-Myc (1:1000, ab32072, Abcam), E-cadherin (1:1000, \#3195, CST), N-cadherin (1:1000, \#13116, CST), Vimentin (1:1000, \#5741, CST), Snail (1:1000, \#3879,
CST), Bax (1:1000, \#14796, CST), cleaved caspase-3 (1: 1000, \#9661, CST), and $\beta$-actin $(1: 10000,66,009-1-\lg$, Proteintech). Next, the membranes were incubated with the indicated secondary antibodies (1:1000, CST) for $1 \mathrm{~h}$. After washing three times, the targeted proteins were visualized using enhanced chemiluminescence (ECL) reagent (Millipore, MA, USA). $\beta$-actin was used as the loading control in this study.

\section{Transwell migration and invasion assays}

Transwell assays were conducted using 24-well plates with chamber inserts with $8.0 \mu \mathrm{m}$ pores (Corning) without (migration) or with (invasion) 2\% Matrigel (BD Science, USA). The cells were digested $24 \mathrm{~h}$ after transfection, and resuspended in serum-free medium. Then, $6 \times 10^{4}$ cells per well were placed into the upper chambers. he lower chamber was filled with $500 \mu \mathrm{l}$ medium with $10 \% \mathrm{FBS}$ as a as a cell nutritional attractant. After incubation at $37^{\circ} \mathrm{C}$ for $24 \mathrm{~h}$, cells in the upper chamber were gently removed with a cotton swab. The migrated or invaded cells were fixed with $4 \%$ polyformaldehyde and visualized by staining with $0.2 \%$ crystal violet for $20 \mathrm{~min}$. Last, the migrated or invaded cells were imaged and quantified by capturing five randomly chosen microscopic fields by using an inverted microscope (Olympus, Japan). All experiments were performed in triplicate.

\section{Three-dimensional (3D) spheroid invasion assays}

3D spheroid invasion assays were carried out as previously reported [19]. The transfected cells were suspended with DMEM containing 10\% FBS $\left(2 \times 10^{4}\right.$ cells/ $\mathrm{mL}$ ), seeded into ultra-low attachment (ULA) roundbottom 96 -well plates $(200 \mu \mathrm{l} /$ well $)$, and cultured 4 days to form tumour spheroids. Next, $100 \mu \mathrm{l} /$ well of DMEM from the spheroid plates was removed and then $100 \mu \mathrm{l}$ of basement membrane matrix (BMM, Corning) was added into each well; the plates were centrifuged at $300 \times \mathrm{g}$ for $3 \mathrm{~min}$ at $4{ }^{\circ} \mathrm{C}$, and incubated at $37^{\circ} \mathrm{C}$ to allow the BMM to solidify. One hour later, $100 \mu$ DMEM containing $10 \%$ FBS was gently added into each well. Final, the spheroids were incubated at $37^{\circ} \mathrm{C}$ in $5 \% \mathrm{CO} 2$, and images were obtained using an inverted microscope (Olympus, Japan). All experiments were carried out in triplicate.

\section{Apoptosis assay}

Apoptosis rates were measured with the Annexin V PE Apoptosis Detection Kit (BD) as reported previously [20]. In brief, the transfected cells were harvested and incubated with binding buffer supplemented with $5 \mu \mathrm{L}$ of Annexin V-PE and $5 \mu \mathrm{L}$ of 7 -AAD for $15 \mathrm{~min}$ in the dark. Finally, apoptosis analysis was performed with a 
FACSCalibur flow cytometer. All experiments were carried out in triplicate.

\section{Fluorescence in situ hybridization (FISH) assay and PDAC TMAs}

In brief, PANC-1 cells were grown in confocal dishes and fixed with $4 \%$ paraformaldehyde prior to permeabilization in PBS with $0.5 \%$ Triton X-100. The Cy3-labelled circEYA3 probes (Geneseed, Shanghai, China) and FAM-labelled miR-1294 probes (GenePharma, Shanghai, China) were diluted, denatured and added to PANC-1 cells at $37^{\circ} \mathrm{C}$ overnight. The next day, cell nuclei were stained with DAPI for $15 \mathrm{~min}$ after hybridization. Finally, images were acquired with a Leica confocal microscope (Leica Microsystems, Germany). Moreover, the expression level of circEYA3 in tissues was evaluated by FISH in TMAs containing samples from 209 PDAC patients who were diagnosed with PDAC at the FUSCC, between 2014 to 2018. All samples were evaluated by two independent pathologists. This study was approved by the Clinical Research Ethics Committee of FUSCC. The intensity of circEYA3 staining was scored as follows: 0 , no staining; 1 , low staining; and 2 , high staining. The percentage of positively stained cells was scored as follows: 0, $0 \%$ (no stained cells); 1,1 $\sim 24 \%$; 2, $25 \sim 49 \%$; 3, $50 \sim 74 \%$; and 4, $75 \sim 100 \%$. The final score was calculated by multiplying the scores for the staining intensity and the percentage of positively stained cells. Subsequently, the samples were divided into two groups: the low expression group (score 0-3) and the high expression group (score 4-8). The sequences of the circEYA3 and miR-1294 probes are listed in Additional file 1: TableS3.

\section{Dual-luciferase reporter assay}

The full-length wild-type (WT) sequence of circEYA3, the c-Myc 3' untranslated region (UTR) and the indicated mutant circEYA3 and c-Myc 3'UTR (Mut) containing the predicted miR-1294 binding sites were separately synthesized and cloned into the dualluciferase reporter vector psi-CHECK-2 (Geneseed, Shanghai, China). The resulting dual-luciferase reporter plasmids (WT or Mut) were co-transfected with the miR-1294 mimic or inhibitor into PANC-1 or MiaPaCa2 cells, respectively, using Lipofectamine 3000 . After 48 $\mathrm{h}$ of incubation, the relative firefly luciferase activities with respect to the corresponding Renilla luciferase activities were measured and analysed using a DualLuciferase Assay System (Promega) following the manufacturer's protocol.

\section{RNA pull-down assay}

Pull-down assays with biotinylated circEYA3 were carried out as previously described [21]. In brief, a biotin- labelled probe specifically targeting circEYA3 and a random oligo probe (RiboBio, Guangzhou, China) were incubated with M280 streptavidin Dynabeads (Invitrogen, USA) at room temperature for $2 \mathrm{~h}$. Lysates of PANC-1 and MiaPaCa-2 cells were incubated with the probe/ bead complexes at $4{ }^{\circ} \mathrm{C}$ overnight. Subsequently, the circEYA3/miRNA/bead complexes were washed three times and eluted from the beads. Then, the enrichment of circEYA3 and related miRNAs in the precipitated complexes was evaluated by qRT-PCR. The sequences used in the pull-down assays are listed in Additional file 1: TableS4.

\section{RNA immunoprecipitation (RIP)}

The RIP assay was conducted using a Magna RIP Kit (Millipore, Billerica, MA, USA) in accordance with the manufacturer's instructions, as described previously [9]. In brief, an anti-Ago2 antibody or negative control immunoglobulin G (IgG; Millipore, USA) was applied for the RIP assay. Transfected cells were harvested and lysed in RNA lysis buffer and were then incubated with $5 \mu \mathrm{g}$ of the anti-Ago 2 antibody or IgG at $4{ }^{\circ} \mathrm{C}$ overnight. Then, $40 \mu \mathrm{L}$ of magnetic beads were added. The next day, the RNA/bead complexes were washed and resuspended in Proteinase $\mathrm{K}$ buffer to separate proteins. Then, the immunoprecipitated RNA was purified and analysed by agarose gel electrophoresis or qRT-PCR. RIP assays were also performed in PANC-1 cells transiently overexpressing miR-1294 and the corresponding negative control.

\section{Measurement of ATP levels}

ATP levels were measured as previously described using an ATP determination kit (Promega; FF2000) in accordance with the manufacturer's instructions [22]. In brief, $1 \times 10^{6}$ cells were mixed with $2.5 \%$ trichloroacetic acid (TCA) to extract ATP from the cell samples. After ATP extraction, the TCA in the sample was diluted to a final concentration of $0.1 \%$ by adding neutralizing Trisacetate buffer $(\mathrm{pH}=7.75)$. Then, $40 \mu \mathrm{L}$ of the diluted sample was added to $100 \mu \mathrm{L}$ of $\mathrm{rL} / \mathrm{L}$ reagent (Promega; FF2000) for ATP measurement using a SpectraMax M5/ M5e Multifunctional Microplate Detection System (Molecular Devices). The ATP standard was continuously diluted to obtain a standard curve for calculating the ATP concentration in each sample. The relative concentrations of ATP were calculated and normalized to those in the control groups. All experiments were performed in triplicate.

\section{Animal experiments}

Animal studies were approved by the Committee on the Ethics of Animal Experiments of Fudan University. To establish xenograft mouse models, a lentiviral vector for 
stable knockdown of circEYA3 was designed based on the si-circEYA3-1 sequences. As mentioned above, the indicated lentiviruses from different groups were harvested and transduced into PANC-1 cells. The human miR-1294 inhibitor plasmid was purchased from Genomeditech (Shanghai, China). Then, the control-shRNA, sh-circEYA3, sh-circEYA3 and the miR-1294 inhibitor were stably transduced into PANC-1 cells and selected with puromycin for 2 weeks. For the tumor growth study, male BALB/c athymic nude mice (4 weeks old) were obtained from Shanghai SLAC Laboratory (Shanghai, China) and inoculated with cells to generate xenograft tumors ( $n=5$ mice/group). Stably transfected PANC-1 cells $\left(3 \times 10^{6}\right)$ in $100 \mu \mathrm{L}$ of PBS were inoculated subcutaneously into the right flanks of the mice. Tumor growth was monitored weekly. Tumors were measured weekly with callipers, and tumor volumes were calculated with the following equation: volume $=0.5 \times$ length $\times$ width $^{2}$. Thirty-eight days after cell inoculation, the body weight were measured, and the tumors were excised, weighed and fixed with $4 \%$ paraformaldehyde for IHC staining.

\section{Statistical analysis}

GraphPad Prism 8.0 and SPSS 23.0 were applied for statistical analysis. Student's t-test was utilized to analyse differences between two groups, and one-way ANOVA was used for multi-group comparisons. The significance of associations between the levels of circEYA3 and miR1294 and clinicopathologic parameters was determined by the $\chi^{2}$ test and Fisher's exact test. Correlations were evaluated by Pearson correlation analysis. Survival was analysed by Kaplan-Meier method, and significance was evaluated with the log-rank test. A $P$-value of $<0.05$ was considered statistically significant for all tests. The results are shown as the mean \pm SD of at least three independent experiments.

\section{Results}

Discovery of oncogenic circRNAs and characterization of circEYA3 in PDAC

To search for potential circRNAs involved in PDAC progression, we systematically analysed the differentially expressed circRNAs in PDAC tissues compared with adjacent noncancerous tissues in two open-access published GEO datasets (GSE79634 and GSE69362) [23, 24]. The volcano plot shows that several circRNAs in GSE79634 and GSE69362 were upregulated or downregulated according to the following criteria: fold change $>$ 2 or $<0.5$ and $P<0.01$ (Fig. 1A). Among the differentially expressed circRNAs, upregulated circRNAs were more common than downregulated circRNAs in GSE69362. In addition, many previous studies have highlighted that some circRNAs are upregulated in
PDAC cells and tissues and perform oncogenic functions in PDAC $[13,25,26]$. Thus, we focused on the upregulated circRNAs in PDAC. The intersection of the upregulated circRNAs in GSE79634 and GSE69362 visualized in a Venn diagram indicated that 13 circRNAs (hsa circ_0064288, hsa_circ_0007367, hsa_circ_0007767, hsa circ_0060733, hsa_circ_0049783, hsa_circ_0092310, hsa circ_0029634, hsa_circ_0007895, hsa_circ_0006117, hsa circ_0074903, hsa_circ_0008253, hsa_circ_0047585, and hsa_circ_0066147) were upregulated in both datasets (Fig. 1B). Subsequently, we selected the 3 most upregulated candidate circRNAs (hsa_circ_0029634, hsa_circ_ 0006117, and hsa_circ_0007895) for further study. Next, the expression levels of these three circRNAs were determined by qRT-PCR in 20 randomly selected pairs of PDAC tissues and matched adjacent normal tissues. The results of qRT-PCR and GEO dataset analysis showed that the level of hsa_circ_0007895 was prominently increased in PDAC tissues compared with adjacent normal tissues, and hsa_circ_0007895 was thus selected for further study (Fig. S1A and S1B). Hsa_circ_0007895 is produced from exons 2-6 of the human EYA3 gene on chromosome 1p35.3 and contains 492 nucleotides (Fig. 1C). Thus, we termed hsa_circ_0007895 "circEYA3" in this article. Next, we observed that the level of circEYA3 was significantly higher in several PDAC cell lines than in the HPDE cell line (Fig. 1D). Among these cell lines, PANC-1 exhibited the highest level and MiaPaCa-2 exhibited the lowest level of circEYA3 (Fig. 1D). Next, to explore the relationship between the level of circEYA3 and the clinical characteristics of PDAC patients, qRTPCR was used to determine the expression of circEYA3 in a cohort comprising 104 pairs of PDAC tumor and matched adjacent normal tissues. As shown in Fig. 1E, circEYA3 was obviously upregulated in the PDAC tissues compared with the matched adjacent normal tissues. Additionally, high expression of circEYA3 was correlated with advanced lymph node invasion and tumour-node-metastasis (TNM) stage (Additional file 1: TableS5). These results indicated that higher circEYA3 expression may be related to the pathogenesis of PDAC. However, no significant correlations were found between the circEYA3 expression level and other features, such as age, sex, tumor grade, tumor location, tumor stage, tumor size, and CA19-9 level.

To prove that circEYA3 is a novel circular rather than linear structure, divergent and convergent primers specific for circEYA3 and the corresponding linear transcript were designed. Then, using cDNA and gDNA from PANC-1 and MiaPaCa-2 cells as templates, we found that circEYA3 was amplified using the divergent primers (an expected 168-bp fragment) only from cDNA; no amplification product was detected from gDNA (Fig. 1F). Next, we conducted Sanger sequencing 


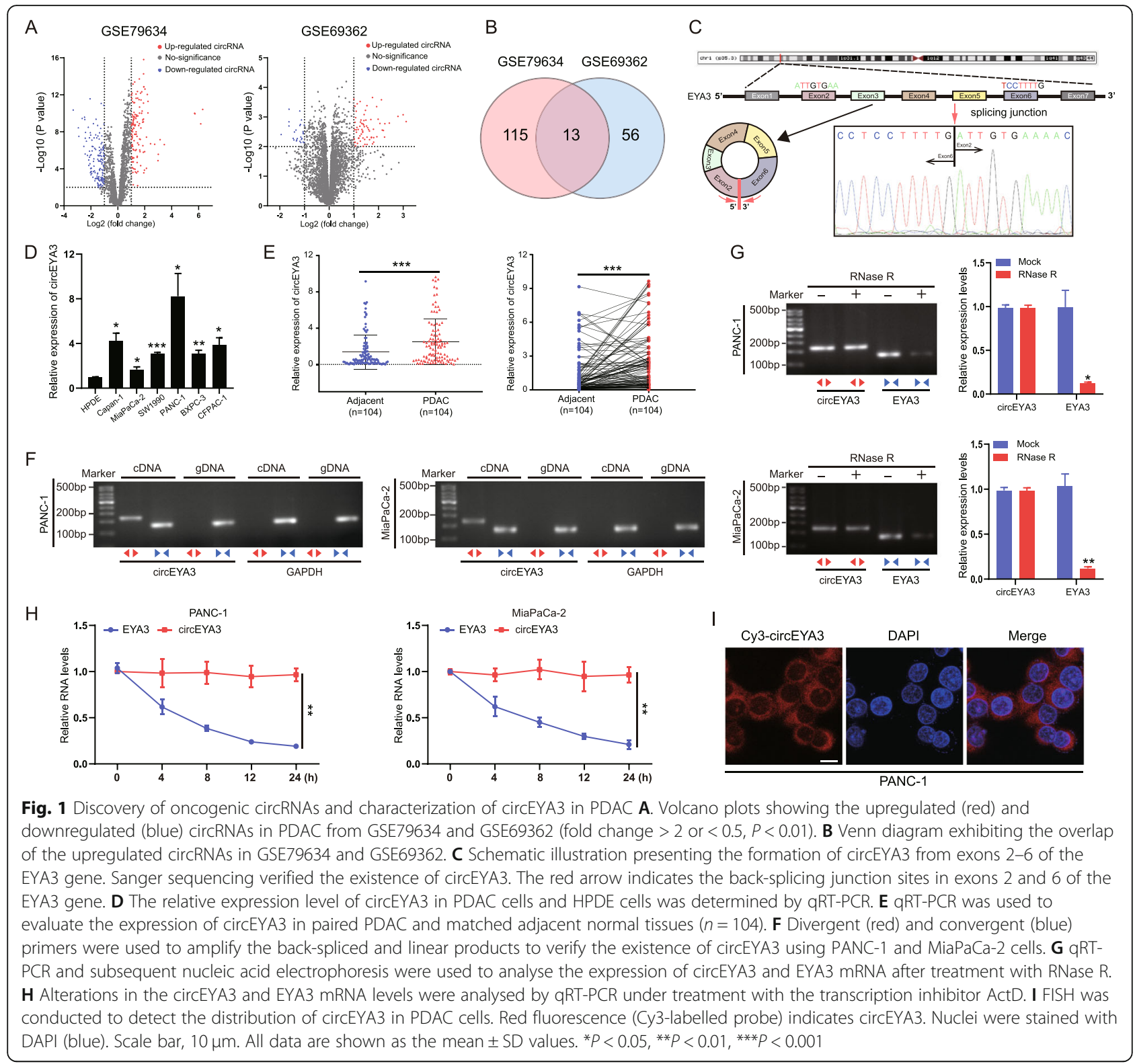

to verify back-splicing sites in the qRT-PCR product of circEYA3 (Fig. 1C). To verify the stability of circEYA3, the extracted RNA was treated with RNase R, and the circular form (circEYA3) was resistant to digestion by RNase $\mathrm{R}$ in both PANC-1 and MiaPaCa- 2 cells; however, the level of the linear form (EYA3 mRNA) was greatly decreased (Fig. 1G). In addition, ActD (a transcription inhibitor) was utilized to confirm the stability of circEYA3, and circEYA3 was found to be more stable than EYA3 mRNA (Fig. 1H). Furthermore, RNA FISH showed that circEYA3 was localized primarily in the cytoplasm (Fig. 1I). Collectively, these data identified circEYA3 as an abundant and stable transcript in PDAC.
CircEYA3 facilitates the proliferation, migration, and invasion and inhibits the apoptosis of PDAC cells in vitro Next, to evaluate the biological roles of circEYA3 in the behaviours of PDAC cells, three siRNAs were designed and constructed to target the back-spliced region of circEYA3 to specifically downregulate its expression (Fig. 2A). After PANC-1 cells were transfected with the three siRNAs, the qRT-PCR results indicated that all three siRNAs effectively silenced circEYA3 without altering the expression of its host linear EYA3 mRNA and that siRNA \#1 and \#2 had the highest knockdown efficiency (Fig. 2B). In addition, circEYA3 was successfully stably overexpressed by transfection of recombinant 


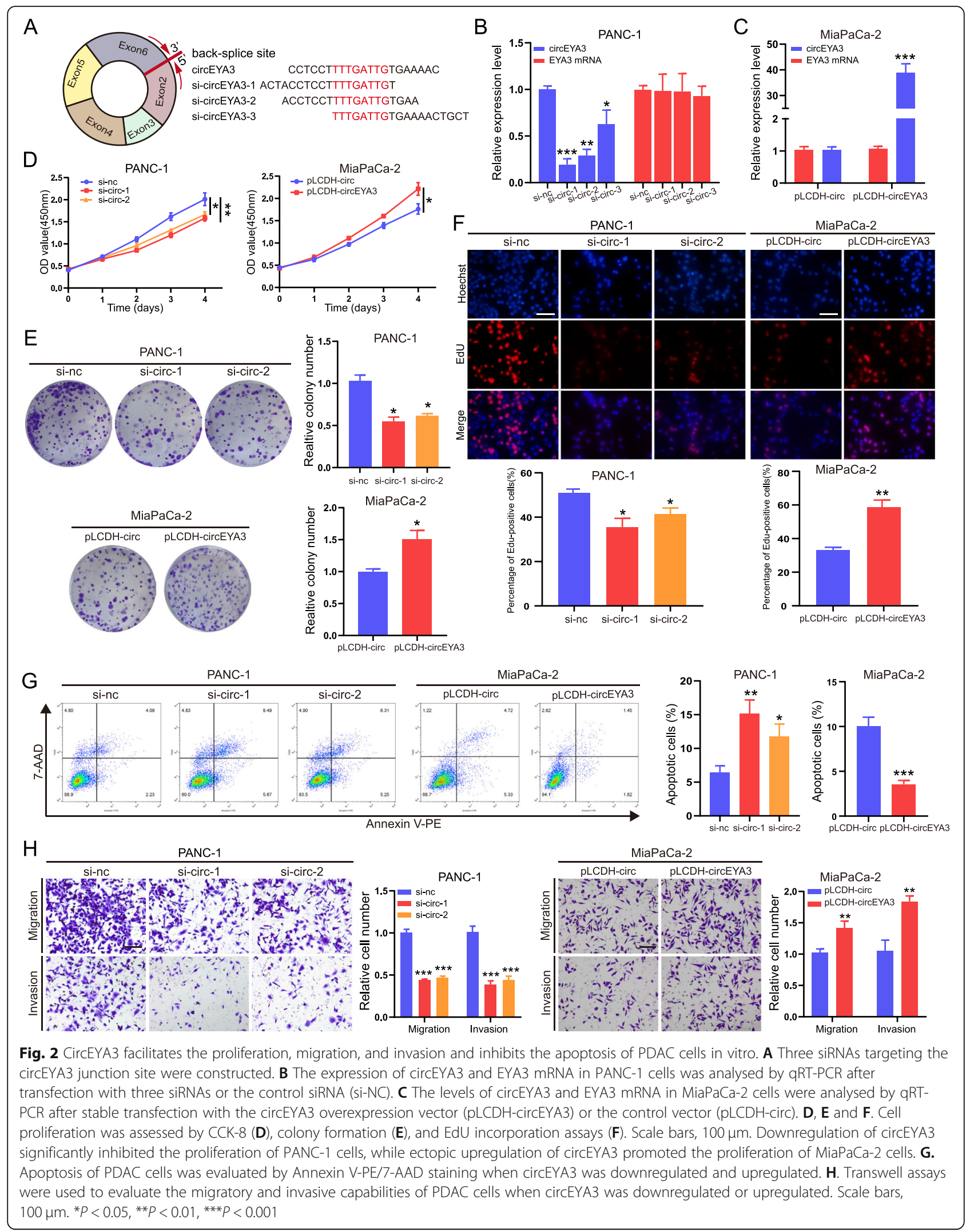


circEYA3 plasmids in MiaPaCa-2 cells, and no significant changes were observed in the level of EYA3 mRNA (Fig. 2C). The CCK-8 assay results showed that circEYA3 knockdown efficiently decreased the proliferation and viability of PANC-1 cells at $96 \mathrm{~h}$ post transfection, while upregulation of the target circEYA3 obviously enhanced the proliferation ability of MiaPaCa-2 cells (Fig. $2 \mathrm{D})$. The colony formation assay further indicated that the number of colonies formed by PANC-1 cells was significantly reduced by knockdown and increased by overexpression of circEYA3 (Fig. 2E). In addition, the EdU incorporation assay as a measure of cell proliferation showed that the percentages of EdU-positive cells were greatly decreased by knockdown but increased by overexpression of circEYA3 (Fig. 2F). To further explore the biological function of circEYA3, apoptosis of PANC-1 and MiaPaCa- 2 cells was evaluated by Annexin V-PE/7AAD staining. The apoptosis rate was increased by downregulation of circEYA3 in PANC-1 cells but decreased by overexpression of circEYA3 in MiaPaCa-2 cells (Fig. 2G). Moreover, the Transwell assay showed that downregulation of circEYA3 obviously decreased the migratory and invasive abilities of PANC- 1 cells, while upregulation of circEYA3 led to the opposite effects in MiaPaCa-2 cells (Fig. 2H). In the 3D spheroid invasion assays, we also observed that downregulation of circEYA3 inhibited tumour spheroid invasion in PANC1 cells, while overexpression of circEYA3 increased spheroid invasion in MiaPaCa-2 cells (Fig. S2A and S2B). Taken together, the results of these experiments indicated that circEYA3 performs an oncogenic function in PDAC cells.

\section{CircEYA3 acts as an efficient miR-1294 sponge in PDAC}

Existing studies have demonstrated that many circRNAs exert their biological effects by sponging miRNAs and subsequently regulating miRNA expression in PDAC $[13,14,25]$. Given that circEYA3 exhibited high stability and was localized mainly in the cytoplasm, as described above, we hypothesized that circEYA3 facilitated malignant biological behaviours of PDAC cells by sponging miRNAs. Therefore, to validate this hypothesis, RIP assays using an anti-Ago2 antibody and IgG were performed in PANC-1 cells and MiaPaCa- 2 cells. We found that endogenous circEYA3 was pulled down by antiAgo2, indicating that circEYA3 bound to miRNAs via the Ago2 protein (Fig. 3A). Subsequently, three databases (starBase, miRanda, and circBank) were used for bioinformatic analysis to predict the potential target miRNAs of circEYA3. Then, 16 candidate miRNAs were identified from the intersection of the miRNAs identified in these three databases, as shown in Fig. 3B. RNA pull-down experiments were subsequently performed using a biotin-labelled probe specific for
circEYA3 and a random oligo negative control probe in PANC-1 and MiaPaCa-2 cells transfected with the pLCDH-circEYA3 overexpression plasmid and the corresponding pLCDH-circ control plasmid. A greater amount of circEYA3 was pulled down in both cell lines after transfection with the pLCDH-circEYA3 overexpression plasmid (Fig. $3 \mathrm{C}$ and D). The expression levels of the 16 candidate miRNAs in the immunoprecipitates were determined using a biotin-labelled probe specific for circEYA3. The qRT-PCR results indicated that miR-1294 was the only miRNA that was statistically abundant in both PANC-1 and MiaPaCa-2 cells, suggesting that miR-1294 specifically interacted with circEYA3 (Fig. 3E). Next, luciferase reporter plasmids containing WT circEYA3 and circEYA3 with a mutated miR-1294 binding site were constructed (Fig. 3F). The luciferase activity of WT circEYA3 was markedly suppressed in PANC-1 cells transfected with the miR-1294 mimic but elevated in MiaPaCa-2 cells transfected with the miR-1294 inhibitor. Conversely, the luciferase activity of the circEYA3 mutant was not significantly changed among the miR-1294 mimic, miR-1294 inhibitor and corresponding negative control groups, indicating the possibility of a direct interaction between miR-1294 and circEYA3 (Fig. 3G). To further validate this interaction, a RIP assay using an anti-AGO2 antibody and negative control IgG was performed in PANC-1 cells transiently overexpressing miR-1294 to pull down circEYA3 and miR-1294, and qRT-PCR was then performed to analyse the levels of circEYA3 and miR-1294 levels in the precipitates. Both circEYA3 and miR-1294 were pulled down in significantly higher quantities by the anti-Ago2 antibody compared with IgG (Fig. $3 \mathrm{H}$ ). Furthermore, circEYA3 and miR-1294 were dramatically more abundant in cells transfected with the miR-1294 mimic compared with cells transfected with the mimic negative control (mimic NC) (Fig. 3H). Next, the expression level of miR-1294 was determined by qRT-PCR in 104 pairs of PDAC tumor and paracarcinoma tissues, and miR-1294 expression was found to be obviously downregulated in PDAC tissues (Fig. 4A). Pearson correlation analysis indicated that miR-1294 expression was negatively associated with circEYA3 expression, as determined by qRT-PCR, in these 104 pairs of fresh frozen PDAC tissues (Fig. 3I). We also found that downregulation of circEYA3 increased miR-1294 expression, while upregulation of circEYA3 decreased miR-1294 expression (Fig. S3A). Furthermore, the FISH results confirmed the co-localization of circEYA3 and miR-1294 mainly in the cytoplasm of PANC-1 cells (Fig. 3L). Taken together, these findings indicated that circEYA3 can directly bind with miR-1294 in PDAC cells. 


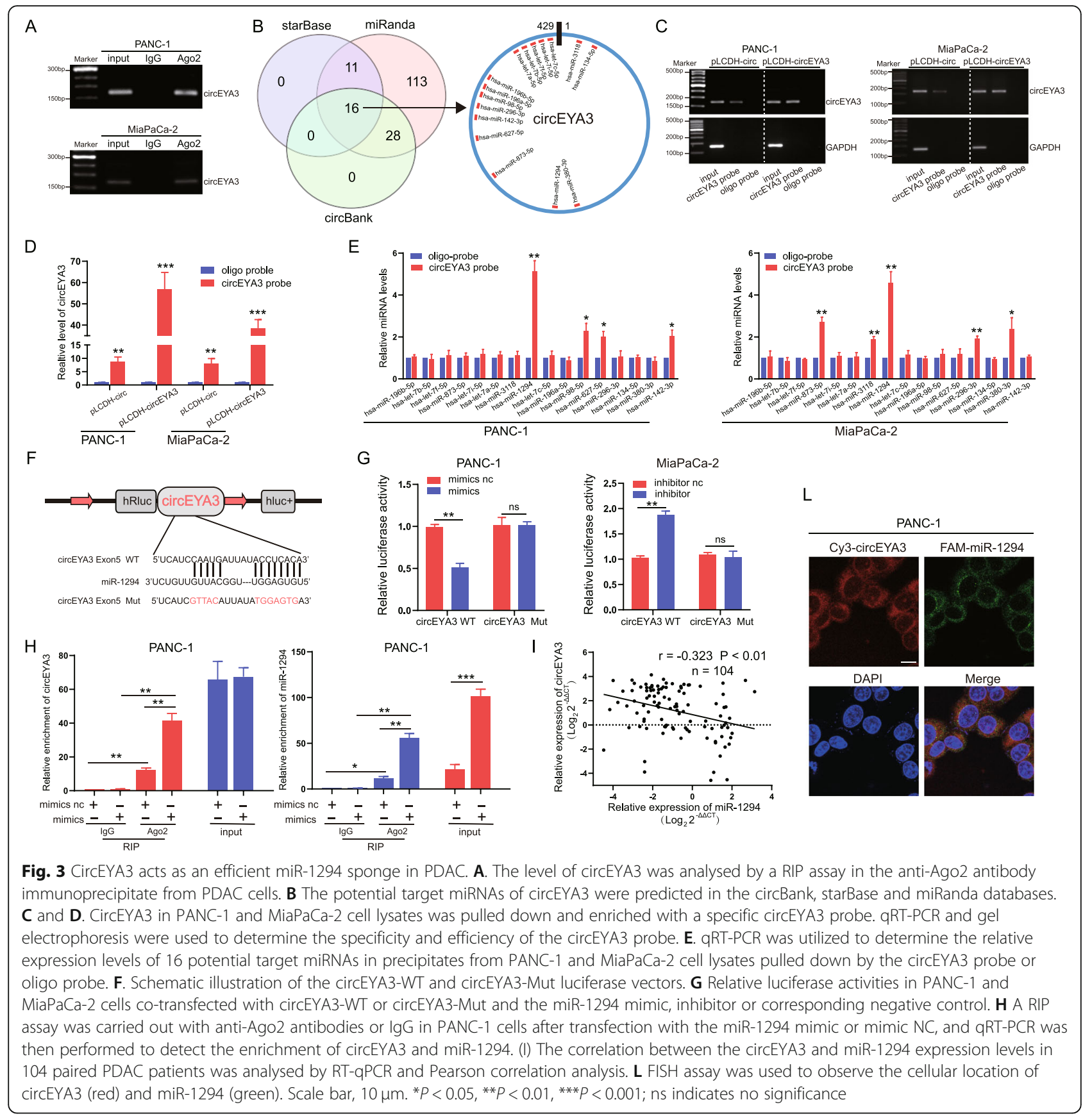

MiR-1294 inhibits the proliferation, migration, and invasion and promotes the apoptosis of PDAC cells in vitro

Reports have confirmed that miR-1294 acts as a tumor suppressor in different cancers [27-29]. A study by Yi et al. also indicated that miR-1294 expression was lower in PDAC tissue samples than in the paired adjacent normal tissues, as determined by qRT-PCR [30], consistent with our analysis of a 104-case cohort of fresh frozen PDAC tissues (Fig. 4A). However, no obvious relationships were observed between miR-1294 expression and
PDAC clinicopathological features based on our qRTPCR results (Additional file 1: Table S5). To determine the specific biological roles of miR-1294 in PDAC, we further explored its functions in PDAC cells. qRT-PCR analysis showed that miR-1294 expression was apparently lower in PDAC cells than in HPDE cells. Among the tested cell lines, PANC-1 exhibited the lowest level and MiaPaCa-2 cells exhibited the highest level of miR1294 (Fig. 4B). Thus, we selected PANC-1 and MiaPaCa-2 cells for further research. PANC-1 and MiaPaCa-2 cells were transfected with the miR-1294 


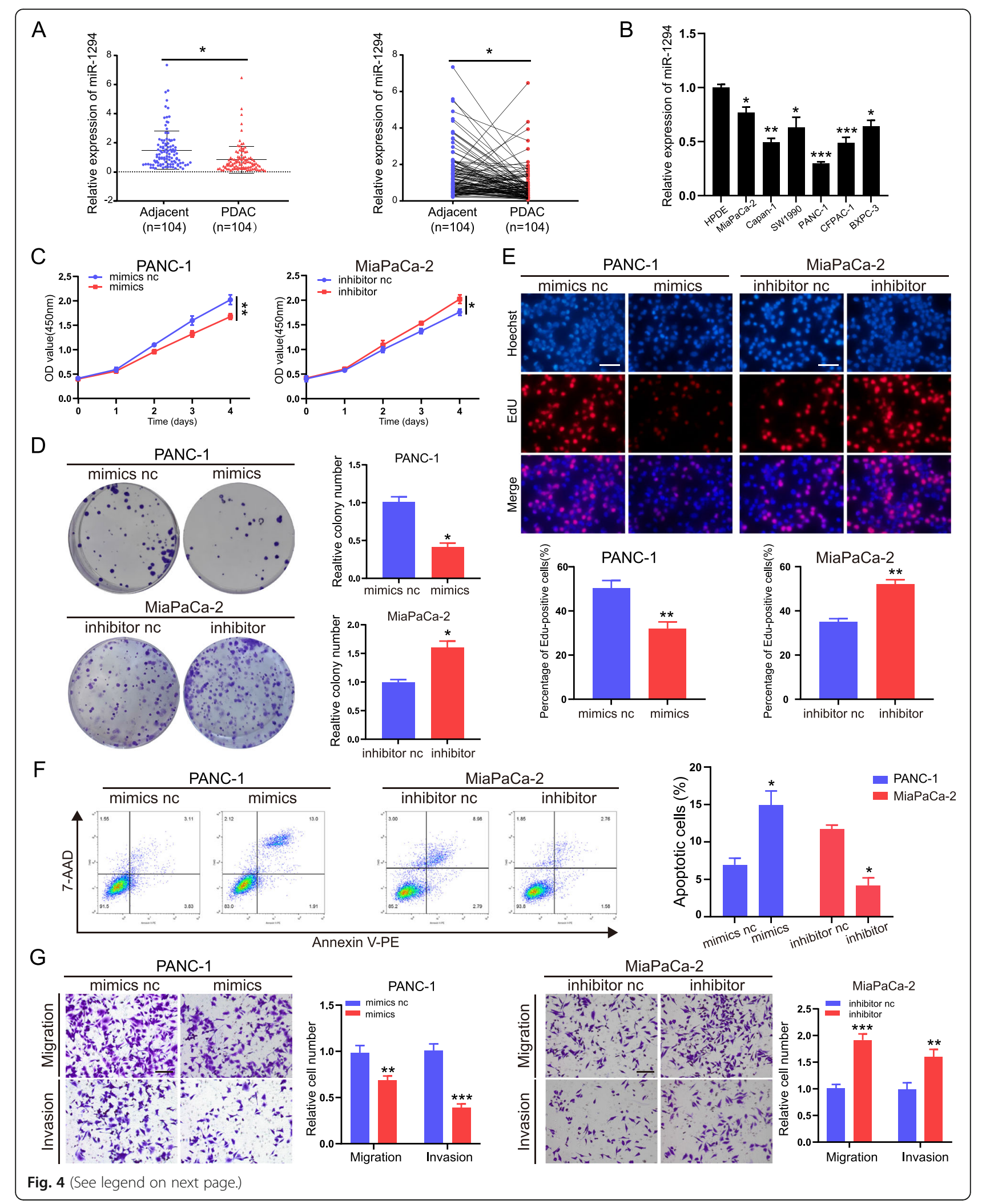


(See figure on previous page.)

Fig. 4 miR-1294 inhibits the proliferation, migration, and invasion and promotes the apoptosis of PDAC cells in vitro. A and B. qRT-PCR was performed to evaluate the expression of miR-1294 in paired PDAC and matched adjacent normal tissues $(n=104)$ (A) and in PDAC cell lines and an HPDE cell line (B). C, D and E. Cell proliferation was assessed by CCK-8 (C), colony formation (D), and EdU incorporation assays (E). Scale bars, $100 \mu \mathrm{m}$. Upregulation of miR-1294 significantly inhibited the proliferation of PANC-1 cells, while ectopic downregulation of circEYA3 promoted the proliferation of MiaPaCa-2 cells. F. Apoptosis of PDAC cells was evaluated by Annexin V-PE/7-AAD staining when miR-1294 was upregulated or downregulated. $\mathbf{H}$. A Transwell assay was performed to evaluate the migratory and invasive capabilities of PDAC cells when miR-1294 was upregulated or downregulated. Scale bars, $100 \mu \mathrm{m} .{ }^{*} P<0.05,{ }^{* *} P<0.01,{ }^{* * *} P<0.001$

mimic or mimic NC or with the miR-1294 inhibitor or inhibitor $\mathrm{NC}$, respectively. Then, the transfection efficiency was confirmed by qRT-PCR (Fig. S3B and S3C). The CCK-8 and colony formation assays showed that upregulated expression of miR-1294 inhibited PANC-1 cell proliferation, whereas downregulated expression of miR-1294 led to the opposite effects (Fig. 4C and D). Consistent with these findings, the EdU incorporation assay showed that the percentage of EdU-positive cells was significantly decreased by upregulation of miR-1294 but increased by downregulation of miR-1294 (Fig. 4E). Moreover, the apoptosis assay showed that upregulation of miR-1294 promoted the apoptosis of PANC-1 cells, whereas downregulation of miR-1294 exerted the opposite effect on MiaPaCa-2 cells (Fig. 4F). The Transwell assay showed that miR-1294 mimic transfection notably decreased the migratory and invasive abilities of PANC1 cells, while miR-1294 inhibitor transfection resulted in the opposite trends in MiaPaCa-2 cells (Fig. 4G). These results demonstrated that miR-1294 may function as a tumor suppressor in PDAC in vitro.

\section{C-Myc is a direct target of miR-1294 in PDAC cells}

To further investigate the downstream genes of miR1294 in PDAC cells, the miRDB, miRWalk, TargetScan and miRTarBase databases were applied to identify the potential downstream target genes of miR-1294. Among the identified potential target genes, two (c-Myc and SURF4) overlapped in the data obtained from these four databases (Fig. 5A). Then, we transfected the miR-1294 mimic and inhibitor into PANC-1 cells and MiaPaCa-2 cells, respectively, to detect whether the levels of the predicted miR-1294 target genes were altered. The qRTPCR results showed that upregulation of miR-1294 decreased the expression of c-Myc in the miR-1294 mimic group but had no effect on the expression of SURF4 (Fig. 5B). Similarly, downregulation of miR-1294 increased the expression of c-Myc (Fig. 5C). In fact, previous studies have proven that miR-1294 can directly target the 3'UTR of c-Myc and function as a tumor suppressor in oesophageal squamous cell carcinoma (ESCC) and oral squamous cell carcinoma (OSCC) [31, 32]. Hence, to validate whether miR-1294 directly targets cMyc in PDAC cells, dual-luciferase reporter assays were performed, and cells co-transfected with c-Myc-WT and the miR-1294 mimics or inhibitor exhibited dramatically reduced or increased luciferase activity, respectively, while the luciferase activity was not significantly altered in cells co-transfected with c-Myc-Mut and the miR1294 mimic or inhibitor (Fig. 5C and D). In line with the roles of miR-1294 in inhibiting PDAC progression, western blot analysis showed that overexpression of miR1294 markedly reduced the expression of c-Myc, Ncadherin, Vimentin, and Snail and elevated the expression of E-cadherin, Bax, and cleaved caspase-3; downregulation of miR-1294 exerted the opposite effects (Fig. 5G). Moreover, in line with the roles of circEYA3 in promoting PDAC progression, downregulation of circEYA3 reduced the expression of c-Myc, N-cadherin, Vimentin, and Snail and elevated the expression of E-cadherin, Bax, and cleaved caspase-3, whereas overexpression of circEYA3 exerted opposite effects (Fig. 5H). Moreover, c-Myc expression was negatively correlated with miR1294 expression in our previous cohort of PDAC tissues (Fig. 5I). The data presented herein indicated that miR1294 can directly bind to the 3' UTR of c-Myc to repress its expression in PDAC cells.

\section{CircEYA3 performs its oncogenic functions by increasing ATP production through the miR-1294/c-Myc axis}

As previously mentioned, altering the expression of circEYA3 significantly affected the expression of c-Myc. To verify whether circEYA3 promoted the progression of PDAC cells through the miR-1294/c-Myc axis, we simultaneously co-transfected si-circEYA3-1 and the miR1294 inhibitor or inhibitor NC into PANC-1 cells and co-transfected the pLCDH-circEYA3 overexpression plasmid and the miR-1294 mimic or mimic NC into MiaPaCa-2 cells for rescue experiments. Western blot analysis and qRT-PCR showed that the downregulation of circEYA3 decreased c-Myc protein expression and that the miR-1294 inhibitor rescued protein expression, while upregulation of circEYA3 and transfection with the miR-1294 mimic caused the opposite effects (Fig. 6A and B). Additionally, circEYA3 expression was positively correlated with c-Myc expression based on our previous qRT-PCR results (Fig. S4A). As shown in Fig. 6D-G, a series of in vitro functional experiments revealed that the biological effects of circEYA3 were reversed by the miR-1294 mimic or inhibitor. The results of the CCK-8 


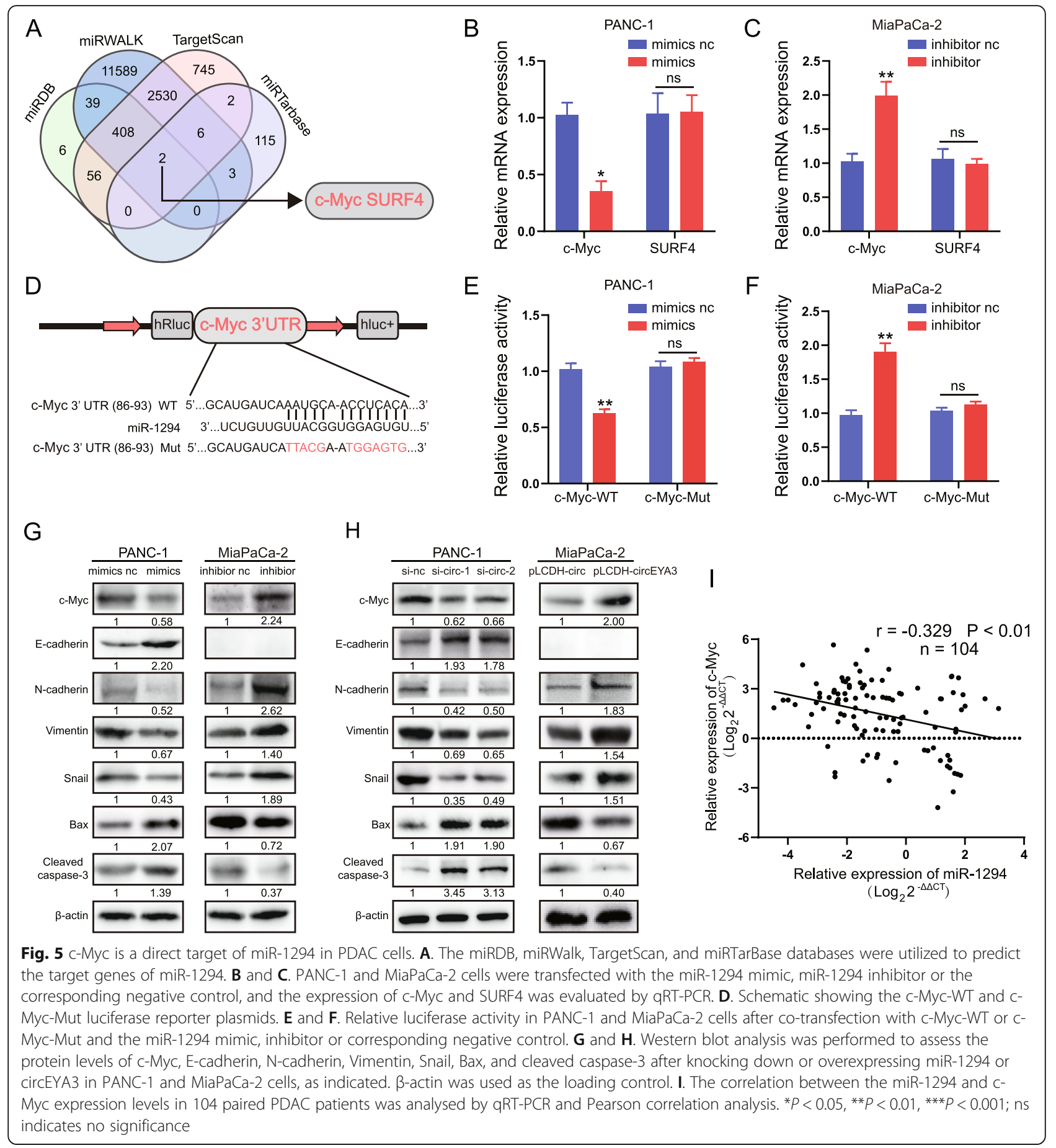

assays, EdU proliferation, colony formation, apoptosis, and Transwell assays showed that the miR-1294 inhibitor reversed the alterations in the biological behaviours of PDAC cells induced by knockdown of circEYA3. Conversely, the miR-1294 mimic offset the alterations in the biological behaviours of PDAC cells induced by overexpression of circEYA3 (Fig. 6D-G). Subsequent western blot analysis also confirmed that the miR-1294 inhibitor and mimic reversed the alterations in EMT- and apoptosis related protein levels caused by alterations in circEYA3 (Fig. 6H and I).

Our previous study confirmed that in pancreatic cancer, c-Myc can increase energy metabolism/ATP production, which is essential for many cellular processes [22]. Therefore, we investigated whether circEYA3 performs its biological functions by affecting the cellular 
A

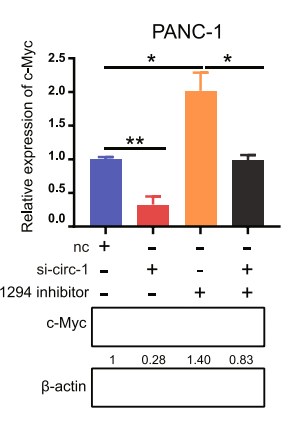

C

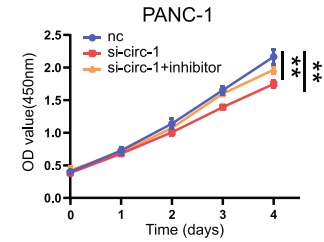

D

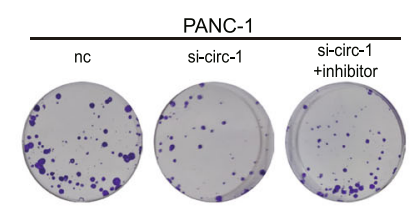

F

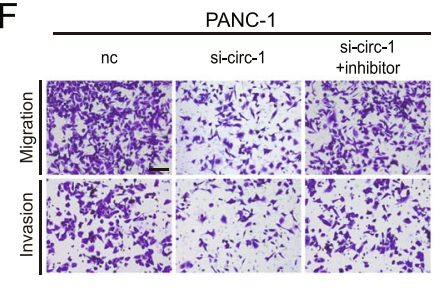

G
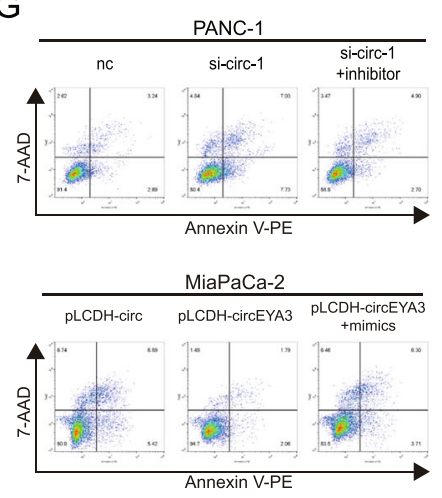

L
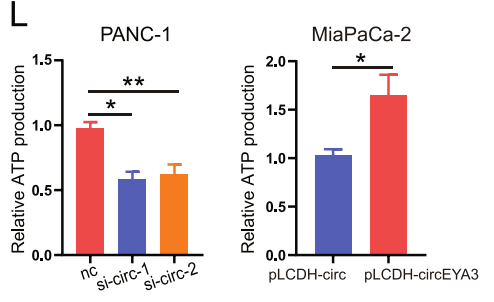

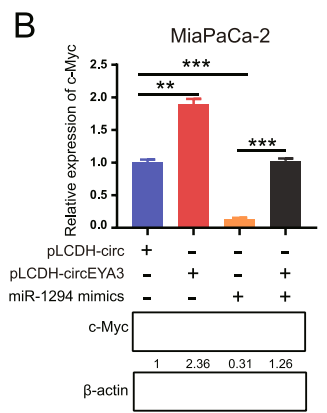

MiaPaCa-2
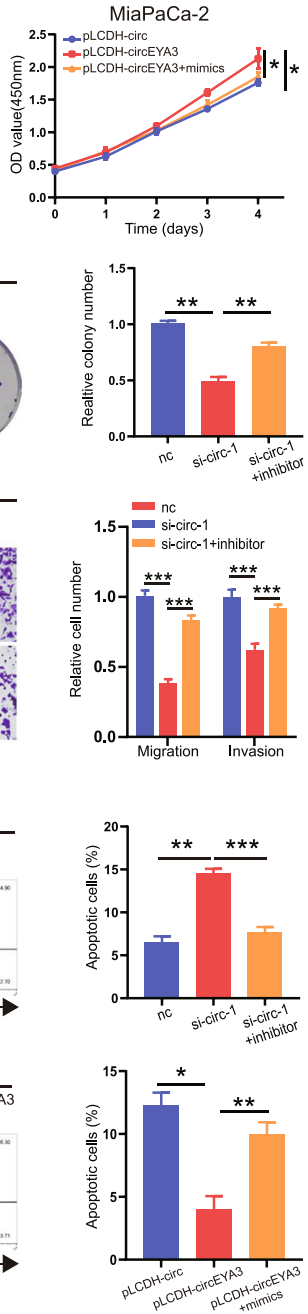

M
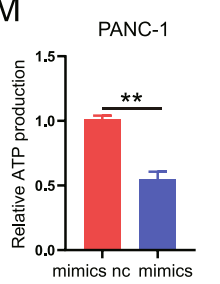
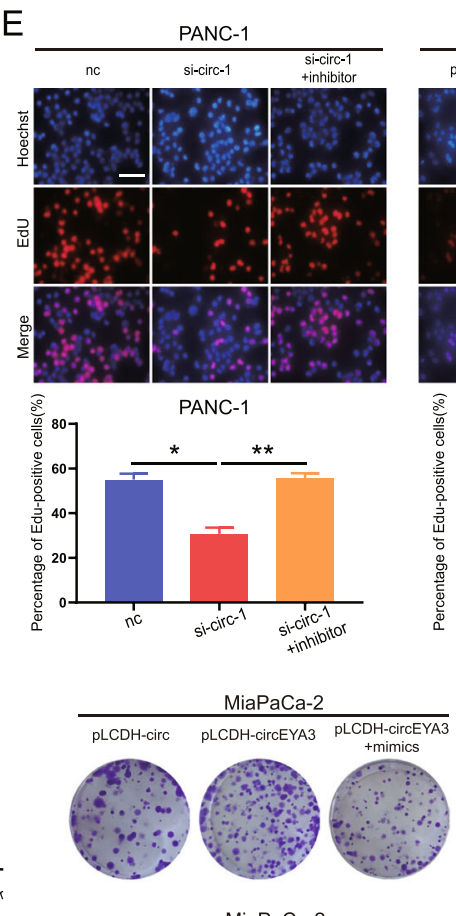

MiaPaCa-2

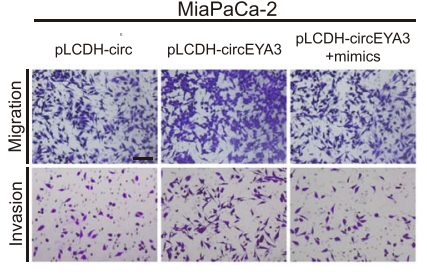

$\mathrm{H}$

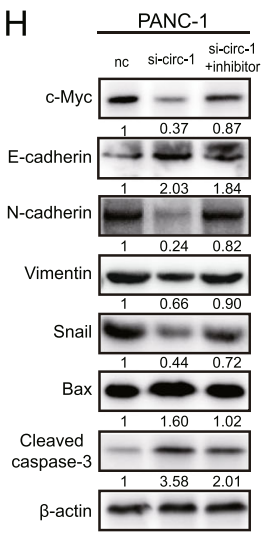

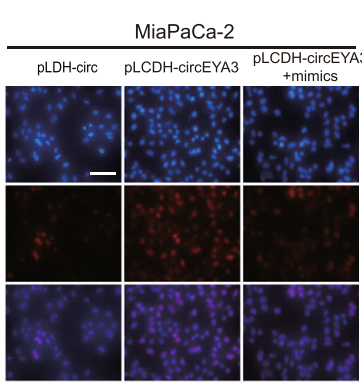
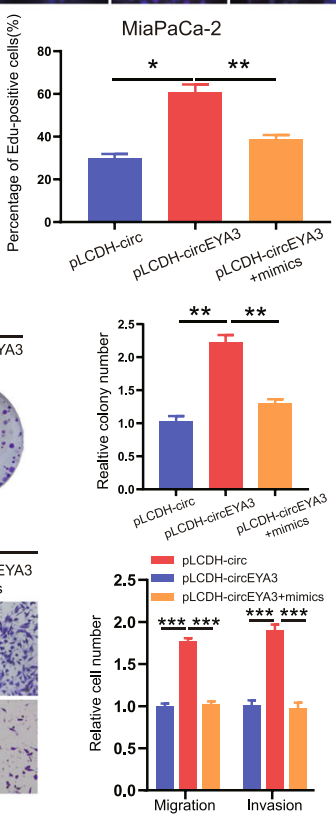

I MiaPaCa-2
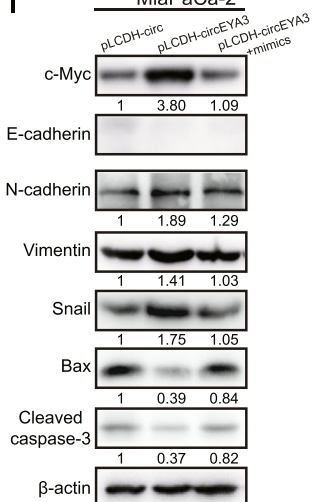
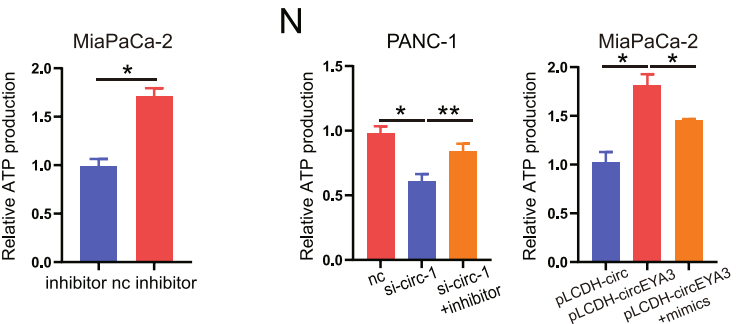

Fig. 6 (See legend on next page.) 


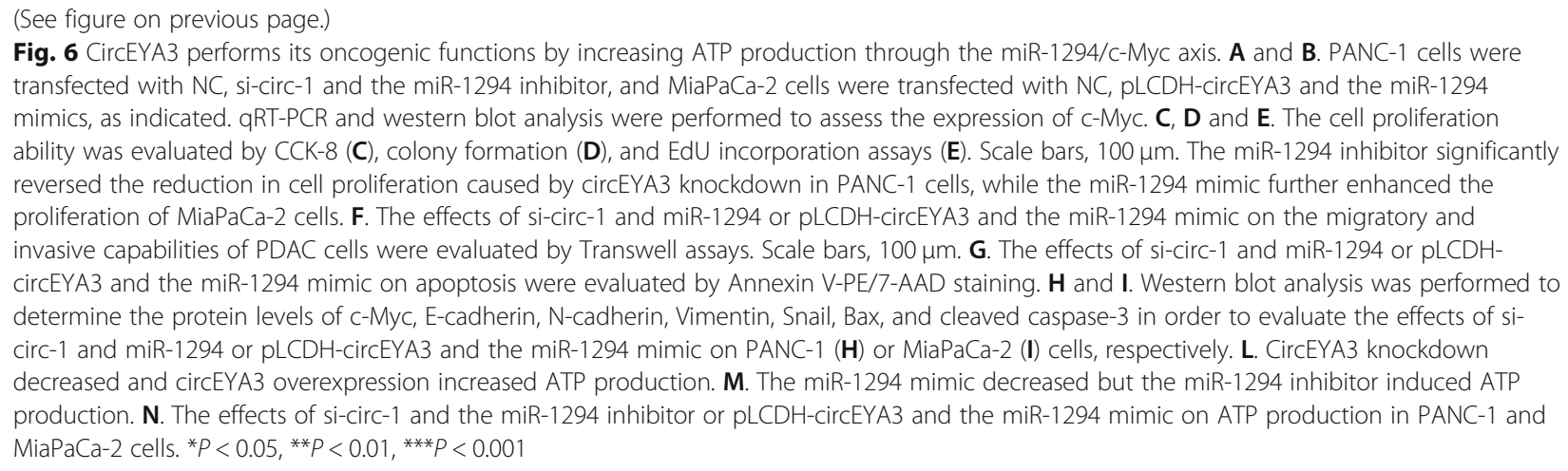

production of ATP. Specifically, downregulation of circEYA3 significantly decreased the production of ATP in PANC-1 cells, while upregulation of circEYA3 exerted the opposite effect in MiaPaCa-2 cells (Fig. 6L). As expected, downregulation of miR-1294 also markedly increased but upregulation of miR-1294 decreased the production of ATP (Fig. 6M). Furthermore, the miR1294 inhibitor and mimic considerably reversed the alterations in ATP production caused by downregulation and upregulation of circEYA3, respectively (Fig. 6N). Specifically, these findings collectively demonstrated that circEYA3 may increase the production of ATP to perform its oncogenic biological functions via the miR1294/c-Myc axis.

\section{Knockdown of circEYA3 suppresses tumor growth from PDAC cells in vivo}

To further explore the potential role of circEYA3 and miR-1294 in vivo, cells were stably transfected with either sh-circ-nc or sh-circEYA3-1 or co-transfected with sh-circEYA3-1 and the miR-1294 inhibitor to establish the xenograft model. The in vivo data confirmed that the tumor volumes and weights were significantly decreased by knockdown of circEYA3 (Fig. 7A and B). Unexpectedly, the miR-1294 inhibitor significantly counteracted the reductions in the tumor volumes and weights induced by knockdown of circEYA3 (Fig. 7A and B). However, there was no significant difference in body weight between the groups (Fig. S4B). Total RNA and protein were extracted from the tumors in each group. Subsequent qRT-PCR showed that circEYA3 knockdown induced major decreases in the mRNA levels of c-Myc and miR-1294 (Fig. 7C). Notably, we observed that the miR-1294 inhibitor largely counteracted this effect (Fig. 7C). Moreover, after knockdown of circEYA3 expression, the protein levels of c-Myc, N-cadherin, Vimentin, and Snail obviously decreased, while those of E-cadherin, Bax and cleaved caspase-3 markedly increased, and the miR-1294 inhibitor partially reversed these alterations (Fig. 7D). Moreover, IHC staining with
anti-c-Myc, anti-E-cadherin, anti-N-cadherin, anti-Bax, anti-cleaved caspase-3, and anti-Ki-67 antibodies was performed on xenograft tumour tissues. The IHC results indicated that circEYA3 knockdown induced decreases in the expression of c-Myc, N-cadherin and Ki-67 but increases in the levels of E-cadherin, Bax and cleaved caspase-3 (Fig. 7E). More importantly, the miR-1294 inhibitor again counteracted these alterations (Fig. 7E). These findings demonstrated that knockdown of circEYA3 inhibits the growth of PDAC tumours in vivo, consistent with the in vitro results.

\section{Overexpression of circEYA3 is associated with poor prognosis in PDAC patients}

To further investigate the clinical significance of circEYA3 in PDAC, FISH was performed on TMAs containing 209 PDAC patients and adjacent normal tissues. The FISH results indicated that circEYA3 expression was markedly upregulated in PDAC tissues compared with adjacent normal tissues (Fig. 8A). Analysis of the clinical characteristics of patients with PDAC revealed that circEYA3 expression was increased with advanced TNM stage (Fig. 8B and C; Table 1). Interestingly, the circEYA3 expression level was significantly correlated with the CA19-9 level; the higher the expression of circEYA3 was, the higher the level of CA19-9 (Table 1). However, no correlations were found between circEYA3 expression and age, sex, tumor location or histological grade (Table 1). Notably, Kaplan-Meier survival analysis revealed that PDAC patients with high circEYA3 expression had poorer clinical outcomes than those with low circEYA3 expression (Fig. 8D). Collectively, these results suggested that overexpression of circEYA3 may predict poor prognosis in PDAC patients.

\section{Discussion}

With the advancement of high-throughput sequencing and bioinformatic techniques, the recognition and understanding of circRNAs has been widely extended. Research has confirmed that circRNAs play regulatory 


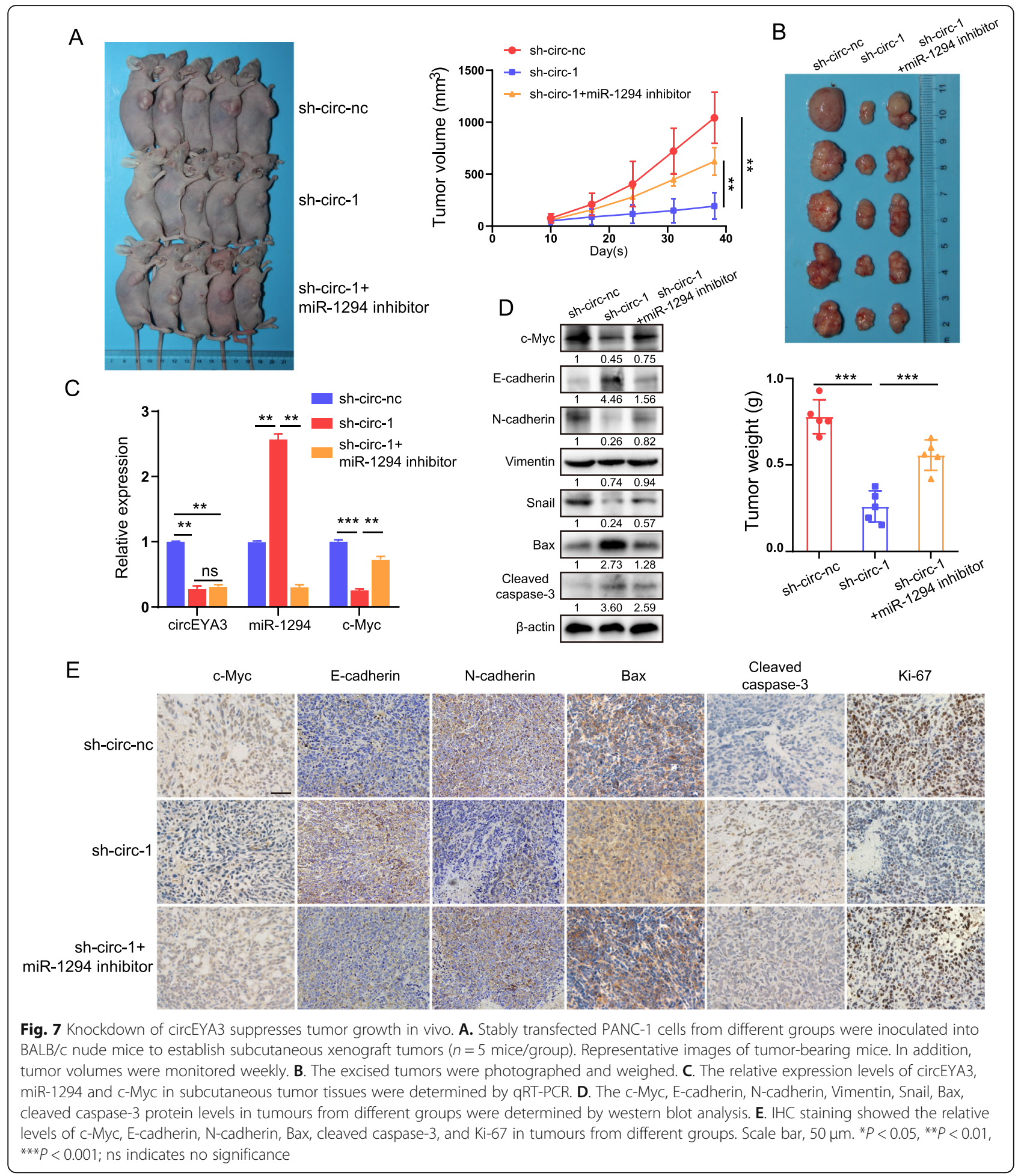

roles in PDAC progression [15, 33]. A previous study demonstrated that circFOKX2 not only interacts with YBX1 and hnRNPK to elevate the expression of the oncogenes NUF2 and PDXK but also functions as a sponge for miR-942 to upregulate the expression of ANK1, GDNF, and PAX6, which contribute to tumor progression in PDAC [25]. Another study demonstrated that hsa_circ_001653 facilitates the progression of PDAC through the miR-37/HOXC6 axis [34]. However, fewer studies have been conducted on circRNAs related to PDAC than on circRNAs related to other gastrointestinal tumors [15]. Through a series of experiments, our 


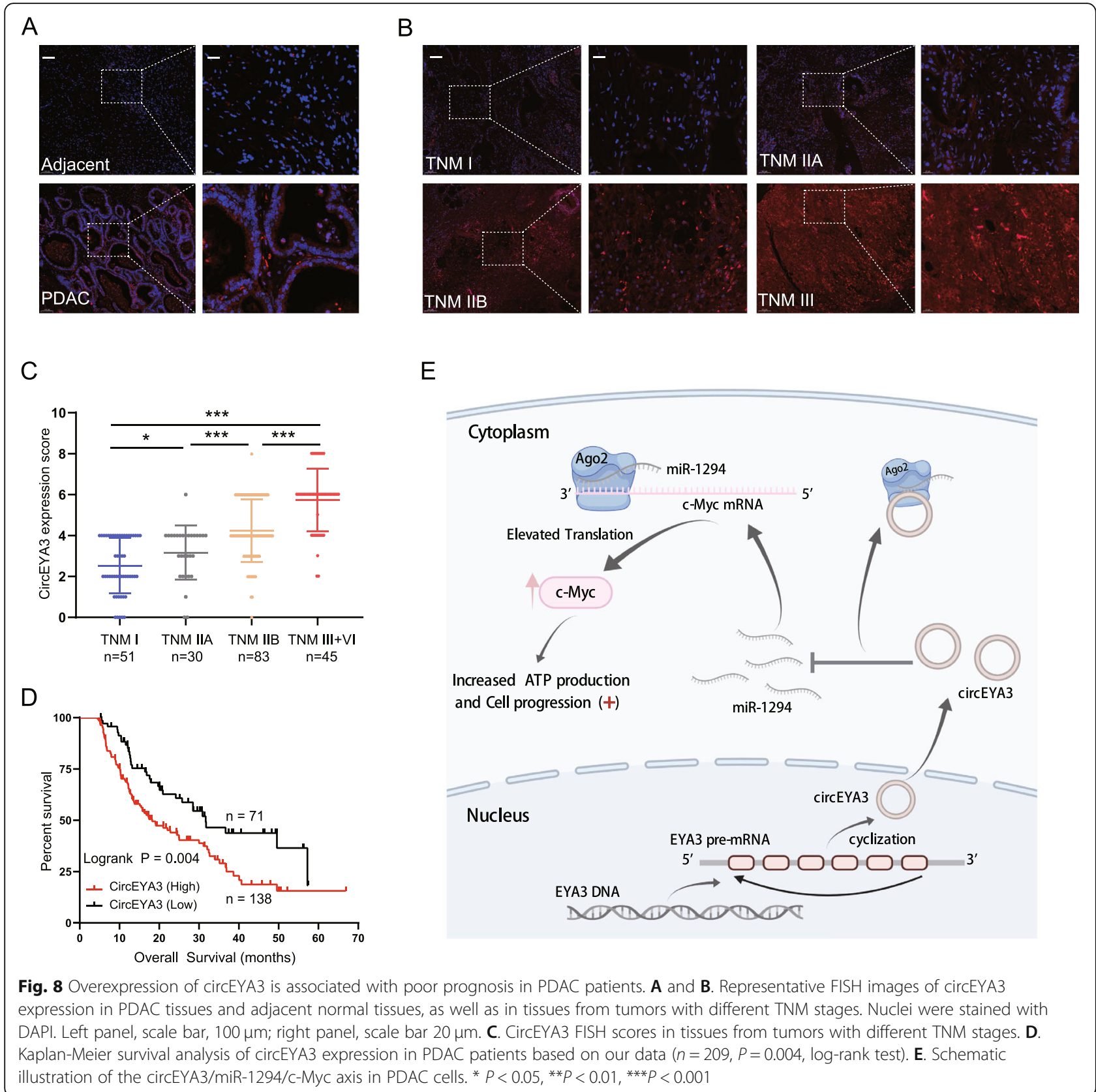

investigation was the first to report the existence of the circEYA3/miR-1294/c-Myc axis in the progression of PDAC (Fig. 8E).

In this study, we first identified differentially expressed circRNAs that were markedly upregulated in PDAC tissues compared with normal tissues in GEO datasets. Many circRNAs have low expression levels in both cancer cells and normal cells [35]. However, based on our preliminary screening of qRT-PCR results for clinical samples, we confirmed that circEYA3 was a circRNA markedly upregulated in PDAC tissues and that a higher expression level of circEYA3 was related to greater lymph node invasion and more advanced tumor stage. CircEYA3 was confirmed through Sanger sequencing to be derived from exons 2-6 of EYA3 by back-splicing, was found to be a highly stable circular transcript under treatment with RNase R and ActD, and was shown to be localized predominantly in the cytoplasm. Moreover, gain- and loss-of-function experiments demonstrated that circEYA3 acted as an oncogene to promote the proliferation, migration and invasion and inhibit the apoptosis of PDAC cells. Additionally, we found that circEYA3 performed its tumor-promoting functions by increasing ATP production in PDAC cells. Furthermore, 
Table 1 Correlation between circEYA3 and clinicopathological parameters in PDAC form TMA $(n=209)$

\begin{tabular}{|c|c|c|c|c|}
\hline \multirow[t]{2}{*}{ Characteristic } & \multirow[t]{2}{*}{ Total (209) } & \multicolumn{2}{|c|}{ circEYA3 expression } & \multirow[t]{2}{*}{$P$ value } \\
\hline & & Low (71) & High (138) & \\
\hline \multicolumn{5}{|l|}{ Age } \\
\hline$\leq 60$ & 94 & 30 & 64 & \\
\hline$>60$ & 115 & 41 & 74 & 0.570 \\
\hline \multicolumn{5}{|l|}{ Sex } \\
\hline Male & 105 & 30 & 75 & \\
\hline Female & 104 & 41 & 63 & 0.098 \\
\hline \multicolumn{5}{|l|}{ Tumor location } \\
\hline Head & 64 & 21 & 43 & \\
\hline Body/tail & 145 & 50 & 95 & 0.814 \\
\hline \multicolumn{5}{|l|}{ Grade } \\
\hline High/Moderate & 142 & 52 & 90 & \\
\hline Low & 67 & 19 & 48 & 0.239 \\
\hline \multicolumn{5}{|l|}{ TNM stage } \\
\hline I & 51 & 32 & 19 & \\
\hline$\| \mathrm{A}$ & 30 & 14 & 16 & \\
\hline$\| \mathrm{B}$ & 83 & 22 & 61 & \\
\hline$|I I+V|$ & 45 & 3 & 42 & $0.000^{* * *}$ \\
\hline \multicolumn{5}{|l|}{ CA19-9 level } \\
\hline$\leq 37 \mathrm{U} / \mathrm{mL}$ & 44 & 25 & 19 & \\
\hline$>37 \mathrm{U} / \mathrm{mL}$ & 165 & 46 & 119 & $0.000^{* * *}$ \\
\hline
\end{tabular}

FISH performed on our PDAC TMAs confirmed that high circEYA3 expression was significantly related to advanced tumor stage and poor patient OS, suggesting that circEYA3 may participate in the progression of PDAC.

CircRNAs play their regulatory roles by acting as miRNA sponges, encoding peptides, interacting with proteins, and regulating transcription or translation [7]. However, circRNAs mostly act as miRNA sponges in cancer cells, resulting in reductions in the expression and function of miRNAs $[8,35]$. One of the well-known and best characterized circRNAs is ciRS-7 (CDR1as), which was shown to contain more than 60 binding sites for miR-7 and to obviously impair the activity of miR-7 in multiple cancer cells where it is highly expressed [3639]. Given that circEYA3 is generated from the coding exons of EYA3 and localized predominantly in the cytoplasm, we hypothesized that circEYA3 may function as miRNA sponge to affect downstream target genes. Hence, we applied bioinformatic tools (circBank, starBase and miRanda) to predict the potential target of circEYA3 and initially identified 16 miRNAs as candidate targets of circEYA3. Notably, we performed a circEYA3 biotin pull-down assay and a RIP assay and confirmed that circEYA3 directly bound to miR-1294. Moreover,
circEYA3 and miR-1294 were confirmed by a dual luciferase reporter assay and FISH to interact with each other and colocalize in the cytoplasm. Further statistical analysis indicated a negative correlation between circEYA3 and miR-1294 expression in PDAC tissues. Abnormal expression of miRNAs is proposed to be associated with proliferation and progression in different human cancers [40]. In fact, miR-1294 has been identified as a downregulated miRNA and a tumor suppressor in many types of cancer [27, 28], including PDAC [30]. Our results are consistent with those of previous studies and proved that miR-1294 is downregulated in both PDAC tissues and cells. Gain- and loss-of-function experiments showed that miR-1294 inhibited the proliferation and progression and decreased the ATP production of PDAC cells, suggesting that miR-1294 functioned as a tumor suppressor in PDAC. More importantly, further rescue experiments both in vivo and in vitro confirmed that miR-1294 reversed the oncogenic effects of circEYA3 in PDAC.

Previous studies have confirmed that miR-1294 inhibits the proliferation and migration of OSCC and ESCC cells by directly binding to the 3' UTR of c-Myc [31,32], a critical oncogene that drives pancreatic cancer tumorigenesis by controlling cell proliferation, metabolism, and apoptosis [16]. In the present study, c-Myc was confirmed to be a target of miR-1294 by a luciferase reporter assay in PDAC cells. We also found that miR1294 overexpression suppressed the activation of c-Myc, indicating that miR-1294 was an important negative regulator of $\mathrm{c}-\mathrm{Myc}$ in PDAC cells. Further statistical analysis showed a marked negative correlation between miR-1294 and c-Myc expression in PDAC tissues. Next, the western blot analysis results showed that altering the expression of circEYA3 greatly affected c-Myc expression both in vitro and in vivo and that miR-1294 reversed this change. The expression of circEYA3 was significantly positively correlated with that of c-Myc, based on our previous qRT-PCR data. Given the particular importance of c-Myc in PDAC progression [17, 22], our findings revealed that circEYA3 acted as a decoy to sequester miR-1294 in order to alleviate the suppression of its target gene c-Myc to in turn increase ATP production in PDAC cells, which can promote the progression of PDAC.

We first proved that circEYA3 promoted PDAC progression by inducing $\mathrm{c}-\mathrm{Myc}$ expression by binding to miR-1294. However, our study has some limitations. First, not all circRNAs can function as miRNA sponges [41]. Whether circEYA3 regulates the proliferation and progression of PDAC cells via other biological processes, such as binding RBPs or being translated into a peptide, requires further investigation. Second, c-Myc is a wellknown oncogenic transcription factor and can bind to 
E-box sequences in the promoters of numerous genes to regulate the expression of downstream genes [16, 42]. According to JASPAR (http://jaspar.genereg.net/), c-Myc shares binding sites with the promoter region of EYA3 (Fig. S5A). The silencing of c-Myc did not affect the level of EYA3 mRNA (Fig. S5B and S5C). Some transcription factors may affect the production of circRNAs $[43,44]$. However, the silencing of c-Myc had no effect on the expression of circEYA3 (Fig. S5B). Thus, further studies are needed to explore the mechanism of upregulation of circEYA3 in PDAC. Our findings partially revealed that $\mathrm{c}-\mathrm{Myc}$ can be regulated by circEYA3 in PDAC, although further in-depth research is needed to explore the more specific relationship between circEYA3 and $\mathrm{c}-\mathrm{Myc}$ in PDAC. Third, our research preliminarily showed that circEYA3 was significantly elevated in PDAC tissues and cells. However, the expression of circEYA3 should be evaluated in a wider range of clinical samples, such as blood, urine, and saliva, to improve its diagnostic and therapeutic value.

\section{Conclusion}

In conclusion, circEYA3 competitively binds miR-1294 to impair the suppressive effect of miR-1294 on c-Myc and increases energy production to facilitate the proliferation and progression of PDAC cells. Our findings expand the understanding of PDAC progression and shed light on therapeutic targets for PDAC.

\section{Abbreviations \\ PDAC: Pancreatic ductal adenocarcinoma; ATCC: American Type Culture Collection; EdU: 5-Ethynyl-2'-deoxyuridine; ADAR1: Adenosine deaminases acting on RNA 1; GLI1: Glioma-associated oncogene 1; CST: Cell Signaling Technology; EMT: Epithelial-to-mesenchymal transition; DMEM: Dulbecco's modified Eagle's medium; YBX1: Y-boxbinding protein; hnRNPK: Heterogeneous nuclear; PDXK: Pyridoxal kinase; NUF2: NUF2 component of NDC80 kinetochore complex.; ANK1: Ankyrin 1; GEO: Gene Expression Omnibus; OS: Overall survival; GDNF: Glial cell-derived neuro- trophic factor; PAX6: Paired box 6; OSCC: Oral squamous cell carcinoma; ESCC: Esophageal squamous cell carcinoma; RBPs: RNA-binding proteins; PVDF: Polyvinylidene fluoride; BCA: Bicinchoninic acid; TMAs: Tissue microarrays}

\section{Supplementary Information}

The online version contains supplementary material available at https:/doi. org/10.1186/s12943-021-01400-z.

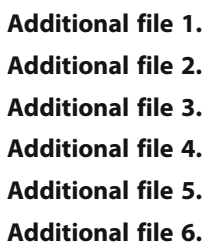

\section{Authors' contributions}

Z.R., S.S., Z.T., and J. X. contributed equally to this work. Z.R., S.S., Z.T., and J. X. performed the experiments. Q.M., J.H., J.L., B.Z., and W.W. analyzed the data and revised the manuscript. C.L., and X.Y. conceived and designed the research. Z.R. wrote the manuscript. The authors read and approved the final manuscript.

\section{Funding}

This study was funded grants from the National Science Foundation for Distinguished Young Scholars of China (No. 81625016), the National Natural Science Foundation of China (No.81902428, 81802352 and 81772555), the Shanghai Sailing Program (No. 19YF1409400), Clinical and Scientific Innovation Project of Shanghai Hospital Development Center (SHDC12018109) and Scientific Innovation Project of Shanghai Education Committee (2019-01-07-00-07-E00057).

\section{Availability of data and materials}

All data that support the conclusion of this study are available from the corresponding authors on reasonable request.

\section{Declarations}

Ethics approval and consent to participate

This research was approved by the the Ethics Committee of FUSCC and Fudan University. All samples were obtained with informed consent.

Consent for publication

All authors confirm their consent for publication the manuscript.

\section{Competing interests}

The authors declare no conflict of interest.

\section{Author details}

'Department of Pancreatic Surgery, Fudan University Shanghai Cancer Center, 270 Dong'An Road, Shanghai 200032, PR China. ${ }^{2}$ Department of Oncology, Shanghai Medical College, Fudan University, Shanghai 200032, China. ${ }^{3}$ Pancreatic Cancer Institute, Fudan University, Shanghai 200032,

China. ${ }^{4}$ Shanghai Pancreatic Cancer Institute, Shanghai 200032, China.

Received: 16 June 2021 Accepted: 3 August 2021

Published online: 21 August 2021

References

1. Paulson AS, Tran Cao HS, Tempero MA, Lowy AM. Therapeutic advances in pancreatic cancer. Gastroenterology. 2013;144(6):1316-26. https://doi.org/1 0.1053/j.gastro.2013.01.078

2. Neoptolemos JP, Kleeff J, Michl P, Costello E, Greenhalf W, Palmer DH. Therapeutic developments in pancreatic cancer: current and future perspectives. Nat Rev Gastroenterol Hepatol. 2018;15(6):333-48. https://doi. org/10.1038/s41575-018-0005-x.

3. Kamisawa T, Wood LD, Itoi T, Takaori K. Pancreatic cancer. Lancet. 2016; 388(10039):73-85. https://doi.org/10.1016/S0140-6736(16)00141-0.

4. Meng $\mathrm{S}$, Zhou $\mathrm{H}$, Feng $Z, X u Z$, Tang $Y$, Li P, et al. CircRNA: functions and properties of a novel potential biomarker for cancer. Mol Cancer. 2017;16(1): 94. https://doi.org/10.1186/s12943-017-0663-2.

5. Memczak S, Jens M, Elefsinioti A, Torti F, Krueger J, Rybak A, et al. Circular RNAs are a large class of animal RNAs with regulatory potency. Nature. 2013:495(7441):333-8. https://doi.org/10.1038/nature11928.

6. Rybak-Wolf A, Stottmeister C, Glažar P, Jens M, Pino N, Giusti S, et al. Circular RNAs in the mammalian brain are highly abundant, conserved, and dynamically expressed. Mol Cell. 2015;58(5):870-85. https://doi.org/10.1016/j. molcel.2015.03.027.

7. Li X, Yang L, Chen LL. The biogenesis, functions, and challenges of circular RNAs. Mol Cell. 2018;71(3):428-42. https://doi.org/10.1016/j.molcel.2018.06. 034.

8. Cheng Z, Yu C, Cui S, Wang H, Jin H, Wang C, et al. circTP63 functions as a ceRNA to promote lung squamous cell carcinoma progression by upregulating FOXM1. Nat Commun. 2019;10(1):3200.

9. Luo Z, Rong Z, Zhang J, Zhu Z, Yu Z, Li T, et al. Circular RNA circCCDC9 acts as a miR-6792-3p sponge to suppress the progression of gastric cancer 
through regulating CAV1 expression. Mol Cancer. 2020;19(1):86. https://doi org/10.1186/s12943-020-01203-8.

10. Jian X, He H, Zhu J, Zhang Q, Zheng Z, Liang X, et al. Hsa_circ_001680 affects the proliferation and migration of CRC and mediates its chemoresistance by regulating BMI1 through miR-340. Mol Cancer. 2020; 19(1):20. https://doi.org/10.1186/s12943-020-1134-8.

11. Shen $S$, Yao T, Xu Y, Zhang D, Fan S, Ma J. CircECE1 activates energy metabolism in osteosarcoma by stabilizing c-Myc. Mol Cancer. 2020;19(1): 151. https://doi.org/10.1186/s12943-020-01269-4.

12. Hanniford D, Ulloa-Morales A, Karz A, Berzoti-Coelho MG, Moubarak RS, Sanchez-Sendra B, et al. Epigenetic silencing of CDR1as drives IGF2BP3mediated melanoma invasion and metastasis. Cancer Cell. 2020;37(1):55-70 e15. https://doi.org/10.1016/j.ccell.2019.12.007.

13. Guo X, Zhou Q, Su D, Luo Y, Fu Z, Huang L, et al. Circular RNA circBFAR promotes the progression of pancreatic ductal adenocarcinoma via the miR-34b-5p/MET/Akt axis. Mol Cancer. 2020;19(1):83. https://doi.org/10.1186/ s12943-020-01196-4.

14. Shen $\mathrm{P}$, Yang $\mathrm{T}$, Chen $\mathrm{Q}$, Yuan $\mathrm{H}, \mathrm{Wu}$ P, Cai B, et al. CircNElL3 regulatory loop promotes pancreatic ductal adenocarcinoma progression via miRNA sponging and A-to-I RNA-editing. Mol Cancer. 2021;20(1):51. https://doi. org/10.1186/s12943-021-01333-7.

15. Rong Z, Xu J, Shi S, Tan Z, Meng Q, Hua J, et al. Circular RNA in pancreatic cancer: a novel avenue for the roles of diagnosis and treatment. Theranostics. 2021;11(6):2755-69. https://doi.org/10.7150/thno.56174.

16. Wirth M, Mahboobi S, Krämer OH, Schneider G. Concepts to target MYC in pancreatic Cancer. Mol Cancer Ther. 2016;15(8):1792-8. https://doi.org/10.11 58/1535-7163.MCT-16-0050

17. Liang C, Shi S, Liu M, Qin Y, Meng Q, Hua J, et al. PIN1 maintains redox balance via the c-Myc/NRF2 Axis to counteract Kras-induced mitochondrial respiratory injury in pancreatic Cancer cells. Cancer Res. 2019;79(1):133-45. https://doi.org/10.1158/0008-5472.CAN-18-1968.

18. Liang C, Shi S, Qin Y, Meng Q, Hua J, Hu Q, et al. Localisation of PGK1 determines metabolic phenotype to balance metastasis and proliferation in patients with SMAD4-negative pancreatic cancer. Gut. 2020;69(5):888-900. https://doi.org/10.1136/gutjnl-2018-317163.

19. Ogawa K, Lin Q, Li L, Bai X, Chen X, Chen H, et al. Aspartate $\beta$-hydroxylase promotes pancreatic ductal adenocarcinoma metastasis through activation of SRC signaling pathway. J Hematol Oncol. 2019;12(1):144. https://doi.org/1 0.1186/s13045-019-0837-z.

20. Meng Q, Liang C, Hua J, Zhang B, Liu J, Zhang Y, et al. A miR-146a-5p/ TRAF6/NF-kB p65 axis regulates pancreatic cancer chemoresistance: functional validation and clinical significance. Theranostics. 2020;10(9):396779. https://doi.org/10.7150/thno.40566.

21. Du WW, Yang W, Liu E, Yang Z, Dhaliwal P, Yang BB. Foxo3 circular RNA retards cell cycle progression via forming ternary complexes with p21 and CDK2. Nucleic Acids Res. 2016;44(6):2846-58. https://doi.org/10.1093/nar/ gkw027.

22. Ji S, Qin Y, Liang C, Huang R, Shi S, Liu J, et al. FBW7 (F-box and WD repeat domain-containing 7) negatively regulates glucose metabolism by targeting the c-Myc/TXNIP (Thioredoxin-binding protein) Axis in pancreatic Cancer. Clin Cancer Res. 2016;22(15):3950-60. https://doi.org/10.1158/1078-0432. CCR-15-2380.

23. Guo $S$, Xu X, Ouyang $Y$, Wang $Y$, Yang J, Yin L, et al. Microarray expression profile analysis of circular RNAs in pancreatic cancer. Mol Med Rep. 2018; 17(6):7661-71. https://doi.org/10.3892/mmr.2018.8827.

24. Li H, Hao X, Wang H, Liu Z, He Y, Pu M, et al. Circular RNA expression profile of pancreatic ductal adenocarcinoma revealed by microarray. Cell Physiol Biochem. 2016;40(6):1334-44. https://doi.org/10.1159/000453186.

25. Wong CH, Lou UK, Li Y, Chan SL, Tong JH, To KF, et al. CircFOXK2 promotes growth and metastasis of pancreatic ductal adenocarcinoma by complexing with RNA-binding proteins and sponging MiR-942. Cancer Res. 2020;80(11): 2138-49. https://doi.org/10.1158/0008-5472.CAN-19-3268.

26. Chen Y, Li Z, Zhang M, Wang B, Ye J, Zhang Y, et al. Circ-ASH2L promotes tumor progression by sponging miR-34a to regulate Notch1 in pancreatic ductal adenocarcinoma. J Exp Clin Cancer Res. 2019;38(1):466. https://doi. org/10.1186/s13046-019-1436-0.

27. Chen H, Liu L, Li X, Shi Y, Liu N. MicroRNA-1294 inhibits the proliferation and enhances the chemosensitivity of glioma to temozolomide via the direct targeting of TPX2. Am J Cancer Res. 2018;8(2):291-301.

28. Zhang ZF, Li GR, Cao CN, Xu Q, Wang GD, Jiang XF. MicroRNA-1294 targets HOXA9 and has a tumor suppressive role in osteosarcoma. Eur Rev Med
Pharmacol Sci. 2018;22(24):8582-8. https://doi.org/10.26355/eurrev_201 812_16621.

29. Pan W, Pang L, Cai HL, Wu Y, Zhang W, Fang JC. MiR-1294 acts as a tumor suppressor in clear cell renal cell carcinoma through targeting HOXA6. Eur Rev Med Pharmacol Sci. 2019;23(9):3719-25. https://doi.org/10.26355/ eurrev_201905_17797.

30. Xu Y, Yao Y, Gao P, Cui Y. Upregulated circular RNA circ_0030235 predicts unfavorable prognosis in pancreatic ductal adenocarcinoma and facilitates cell progression by sponging miR-1253 and miR-1294. Biochem Biophys Res Commun. 2019;509(1):138-42. https://doi.org/10.1016/j.bbrc.2018.12.088.

31. Liu K, Li L, Rusidanmu A, Wang Y, Lv X. Down-regulation of MiR-1294 is related to dismal prognosis of patients with esophageal squamous cell carcinoma through elevating C-MYC expression. Cell Physiol Biochem. 2015; 36(1):100-10. https://doi.org/10.1159/000374056.

32. Wang Z, Yan J, Zou T, Gao H. MicroRNA-1294 inhibited oral squamous cell carcinoma growth by targeting c-Myc. Oncol Lett. 2018;16(2):2243-50. https://doi.org/10.3892/ol.2018.8967.

33. Wang $Y Z$, An $Y, L i B Q$, Lu J, Guo JC. Research progress on circularRNAs in pancreatic cancer: emerging but promising. Cancer Biol Ther. 2019;20(9): 1163-71. https://doi.org/10.1080/15384047.2019.1617563.

34. Shi H, Li H, Zhen T, Dong Y, Pei X, Zhang X. hsa_circ_001653 Implicates in the Development of Pancreatic Ductal Adenocarcinoma by Regulating MicroRNA-377-Mediated HOXC6 Axis. Mol Ther Nucleic Acids. 2020;20:25264.

35. Goodall GJ, Wickramasinghe VO. RNA in cancer. Nat Rev Cancer. 2021;21(1): 22-36. https://doi.org/10.1038/s41568-020-00306-0.

36. Li RC, Ke S, Meng FK, Lu J, Zou XJ, He ZG, et al. CiRS-7 promotes growth and metastasis of esophageal squamous cell carcinoma via regulation of miR-7/HOXB13. Cell Death Dis. 2018;9(8):838. https://doi.org/10.1038/s41419018-0852-y.

37. Zhang J, Hu H, Zhao Y, Zhao Y. CDR1as is overexpressed in laryngeal squamous cell carcinoma to promote the tumour's progression via miR-7 signals. Cell Prolif. 2018;51(6):e12521. https://doi.org/10.1111/cpr.12521.

38. Jian F, Yangyang $R$, Wei $X$, Jiadan $X, N a L$, Peng $Y$, et al. The prognostic and predictive significance of circRNA CDR1as in tumor progression. Front Oncol. 2020;10:549982.

39. Kristensen LS, Ebbesen KK, Sokol M, Jakobsen T, Korsgaard U, Eriksen AC, et al. Spatial expression analyses of the putative oncogene ciRS-7 in cancer reshape the microRNA sponge theory. Nat Commun. 2020;11(1):4551. https://doi.org/10.1038/s41467-020-18355-2.

40. Harrandah AM, Mora RA, Chan EKL. Emerging microRNAs in cancer diagnosis, progression, and immune surveillance. Cancer Lett. 2018;438:12632. https://doi.org/10.1016/j.canlet.2018.09.019.

41. Jeck WR, Sharpless NE. Detecting and characterizing circular RNAs. Nat Biotechnol. 2014;32(5):453-61. https://doi.org/10.1038/nbt.2890.

42. Chang TM, Chu PY, Hung WC, Shan YS, Lin HY, Huang KW, et al. C-Myc promotes lymphatic metastasis of pancreatic neuroendocrine tumor through VEGFC upregulation. Cancer Sci. 2021;112(1):243-53. https://doi. org/10.1111/cas.14717.

43. Yang Z, Qu CB, Zhang Y, Zhang WF, Wang DD, Gao CC, et al. Dysregulation of p53-RBM25-mediated circAMOTL1L biogenesis contributes to prostate cancer progression through the circAMOTL1L-miR-193a-5p-Pcdha pathway. Oncogene. 2019;38(14):2516-32. https://doi.org/10.1038/s41388-018-0602-8.

44. Patop IL, Wüst S, Kadener S. Past, present, and future of circRNAs. EMBO J. 2019;38(16):e100836. https://doi.org/10.15252/embj.2018100836.

\section{Publisher's Note}

Springer Nature remains neutral with regard to jurisdictional claims in published maps and institutional affiliations. 